\title{
New imaging techniques for the evaluation of gastrointestinal diseases
}

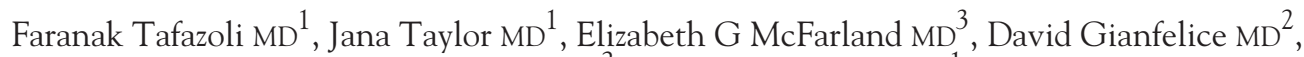 \\ Luigi Lepanto $\mathrm{MD}^{2}$, Caroline Reinhold $\mathrm{MD}^{1}$
}

F Tafazoli, J Taylor, D Gianfelice, EG McFarland, L Lepanto, $\mathrm{C}$ Reinhold. New imaging techniques for the evaluation of gastrointestinal diseases. Can J Gastroenterol 2000;14(Suppl D):163D-180D. This article provides an overview of recently developed, noninvasive imaging modalities for the evaluation of gastrointestinal disease processes. The advent of spiral computed tomography, magnetic resonance cholangiopancreatography and conventional magnetic resonance imaging has facilitated the noninvasive assessment of pancreaticobiliary disease. Magnetic resonance cholangiopancreatography provides projectional images of the biliary tree and pancreatic duct, similar to those achieved by direct cholangiography, without the need to administer contrast medium. Spiral computed tomographic colonography provides virtual colonoscopic images of the colonic mucosa, allowing the detection of polyps without the risk associated with colonoscopy.

Key Words: Gastrointestinal disease; Magnetic resonance cholangiopancreatography; Magnetic resonance imaging; Spiral computed tomography

\section{Nouvelles techniques d'imagerie pour l'évaluation des maladies gastro-intestinales}

RÉSUMÉ : Le présent article donne un aperçu des nouvelles techniques d'imagerie, non effractives, pour l'évaluation des maladies gastro-intestinales. L'arrivée de la tomodensitométrie hélicoïdale, de la cholangio-pancréatographie par résonance magnétique et de l'imagerie usuelle par résonance magnétique a facilité l'évaluation non effractive des maladies pancréato-biliaires. La cholangio-pancréatographie par résonance magnétique fournit des images de projection de l'arbre biliaire et du canal pancréatique, semblables à celles obtenues par la cholangiographie directe sans l'administration d'une substance de contraste. La colographie par tomodensitométrie hélicoïdale fournit des images virtuelles de la muqueuse du côlon, ce qui permet la détection de polypes mais sans les risques associés à la coloscopie.
W ith the advent of newer imaging techniques, the number of noninvasive diagnostic modalities available to the clinician for evaluating patients with suspected gastrointestinal disorders has increased considerably. An overview of a number of these new imaging modalities for the evaluation of gastrointestinal disease processes is provided. The availability of spiral computed tomography (CT), magnetic resonance cholangiopancreatography (MRCP) and conventional magnetic resonance imaging (MRI) has facilitated the noninvasive assessment of pancreaticobiliary disease. MRCP provides projectional images of the biliary tree and pancreatic duct, similar to direct cholangiopancreatography, without the need for contrast medium injection. In addition, the role of spiral CT colonography (virtual colonoscopy), which provides a three-dimensional simulated visualization of the colonic mucosa, is reviewed.

\section{SPIRAL CT APPLICATIONS FOR HEPATOBILIARY IMAGING}

Introduction to spiral CT imaging: With recent technological developments and the refinement of slip ring scanning, spiral CT imaging has become the preferred method of

\footnotetext{
${ }^{1}$ Department of Diagnostic Radiology, University of McGill and ${ }^{2}$ Department of Diagnostic Radiology, University of Montreal, Montreal , Quebec ${ }^{2}$; ${ }^{3}$ Mallinckrodt Institute of Radiology, University of Washington, St Louis, Missouri, USA

Correspondence and reprints: Dr Caroline Reinhold, Department of Radiology, Montreal General Hospital, 1650 Cedar Avenue, Montreal, Quebec H3G 1A4. Telephone 514-937-6011 ext 2872, fax 514-934-8263, e-mail reinhold@rad.mgh.mcgill.ca Received for publication March 4, 1999. Accepted March 12, 1999
} 

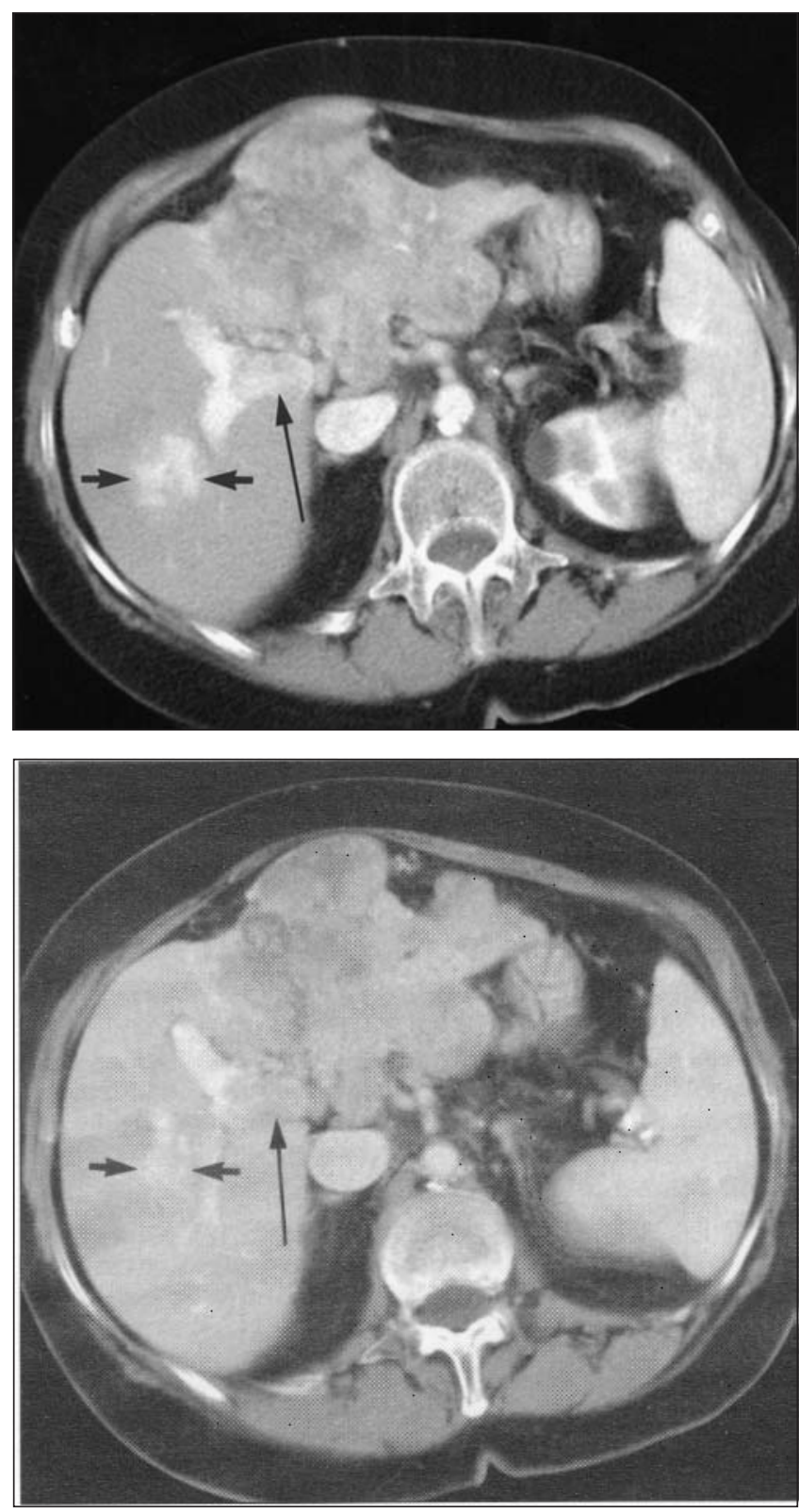

Figure 1) Spiral computed tomographic arterial phase imaging in a patient with multicentric hepatoma involving the left and right lobes. Images obtained late in the arterial phase (top) show the enhancing lesion (arrows) in the right lobe. The right lobe lesion (arrows) is less evident on the portal phase images (bottom) and may be interpreted as a vascular defect. Note the portal vein thrombosis (long arrow)

obtaining diagnostic CT images of the hepatobiliary system $(1,2)$. Slip ring scanning allows continuous rotation of the $\mathrm{x}$-ray tube situated in the scanner gantry and, hence, allows a rapid acquisition of large volumes of information compared with that obtained with classic single slice CT imaging techniques. Several important advantages result from this new scanning method, namely increased speed of acquiring imaging; virtual elimination of 'unscanned' areas due to variations in patient respiration; optimal use and timing of intravenous contrast administration; and acquisition of a volume of data that can more efficiently be converted to three-dimensional imaging for subsequent surgical and therapeutic planning $(2,3)$.

With the advent of increased speed and flexibility, image acquisition can be achieved in a region of interest during a single breath-hold (20 to $30 \mathrm{~s}$ ). Optimal timing of image acquisition also allows studies to be obtained in the different phases of peak arterial and venous contrast enhancement periods. This capacity allows increased lesion detection, and better visualization of arterial and venous structures in tumour encasement, with the added benefit of shorter imaging studies for the patient.

Hepatobiliary imaging - Liver imaging: Lesion detection is the most frequent clinical indication for CT evaluation of liver parenchyma. Other indications, including lesion characterization and evaluation of perfusion disorders, are under clinical investigation and are beyond the scope of this article.

Conventional CT imaging and spiral CT protocols capitalize on the fact that most hepatic focal lesions are hypovascular compared with the normal liver parenchyma. Injection of iodinated contrast and scanning at peak enhancement of normal parenchyma accentuate this difference in vascularity with pathological lesions. Spiral imaging protocols offer several distinct advantages over classical CT techniques with the capacity to acquire a full examination of liver parenchyma in both peak arterial (approximately $20 \mathrm{~s}$ after injection of contrast) and venous enhancement phases (70 s after injection) in less time required for a single examination with the single slice method $(4,5)$.

Arterial phase images offer the distinct advantage of demonstrating early arterial enhancement in certain less common lesions that receive their vascularity from the hepatic arteries directly (ie, carcinoid metastases, focal nodular hyperplasia and some hepatomas) (Figure 1). Images acquired in the venous phase with spiral protocols closely resemble standard slice by slice CT images with two distinct differences: all images in spiral CT protocols are obtained in the peak 'enhancement window' (hence optimal distinction between normal parenchyma and focal lesion) and, secondarily, volume spiral acquisition virtually eliminates the possibility of 'unscanned' portions of the liver due to variations in patient respiration.

Spiral CT arterial portography: CT images acquired during arterial portography studies have proved to be superior in their ability to detect focal hepatic lesions (Figure 2). The main indication for these more invasive studies is the evaluation of liver parenchyma in patients with known localized hepatic metastatic disease who may be candidates for curative resection $(6,7)$. Injection of iodinated contrast through a catheter placed in either the hepatic or splenic artery allows more intense enhancement of normal liver parenchyma and, hence, increased visibility of pathological nonvascular focal lesions. Spiral CT rapid acquisition allows all of the liver parenchyma to be scanned in this short 'peak enhancement window', as opposed to slice by slice protocols, where the last images may be acquired well past the peak and into the equilibrium phase of contrast enhancement. 


\section{COPYRIGHT PULSUS GROUP INC, - DO NOT COPY
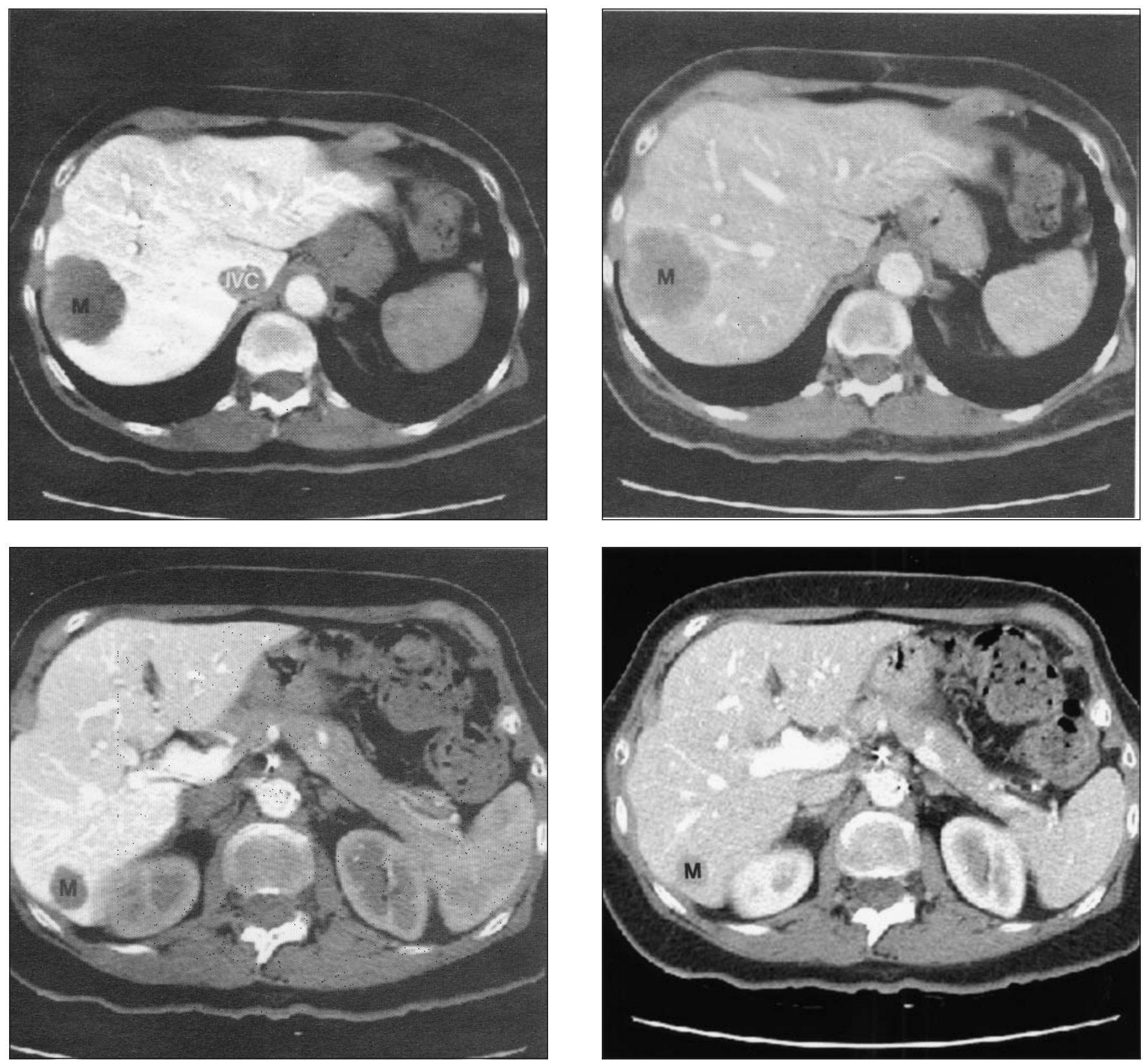

Figure 2) Top left, bottom left Spiral computed tomographic arterial portograph showing metastases (M) in segments 7 and 6, respectively. Top right, bottom right Images obtained later, during the equivalent of the portal venous phase of an intravenous infusion. Note how the metastases (M) are less well demarcated from normal liver parenchyma. IVC Inferior vena cava

Volume data acquisition and three-dimensional applications: The objective of image processing and threedimensional reconstruction is to obtain the greatest amount of information possible from the data acquired by spiral CT. The three-dimensional images augment the diagnostic content of the data set compared with transverse plane images alone and can obviate the need for more invasive procedures.

Short of attaining isotropic imaging and true three-dimensional volume acquisition, spiral CT allows the acquired image data to be treated as a volume data set. Volume rendering techniques can then be used to portray images from a perspective other than the traditional two-dimensional transverse plane views. Several features of spiral CT are instrumental in allowing three-dimensional imaging. The continuous acquisition of image data is important in yielding a volume data set, as is the speed of acquisition. This allows the entire data set to be obtained during a single breath-hold - a prerequisite for valid three-dimensional reconstruction. In addition, the increased speed of acquisition allows data to be obtained during different phases of contrast administration. It is possible to synchronize acquisition with the precise occurrence of arterial and portal enhancement, leading to exquisite visualization of the different vascular structures $(8,9)$. These features, coupled with the increased computing power available for postprocessing 


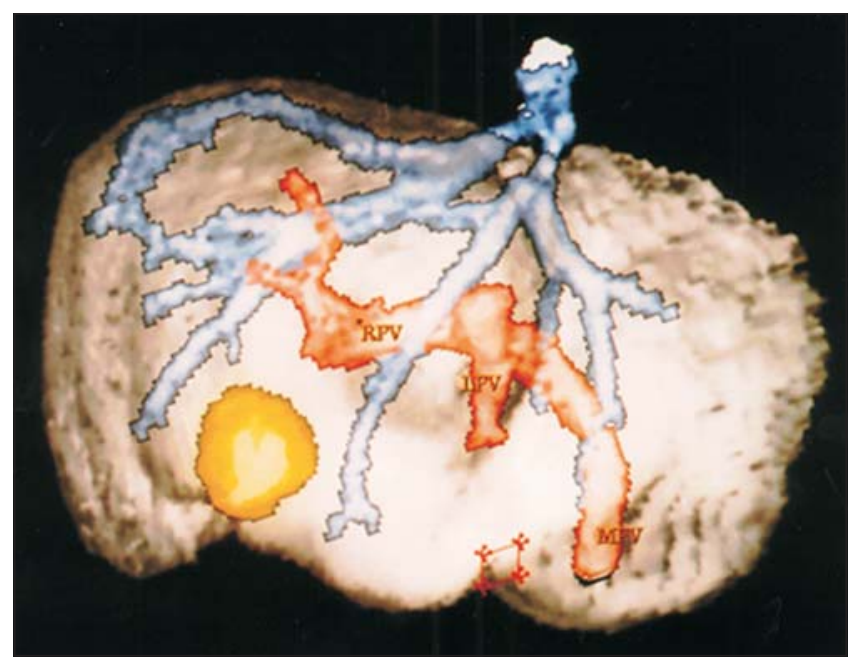

Figure 3) Spiral computed tomographic three-dimensional reconstruction showing the location of the metastatic lesion (segment 5 , encoded in yellow) in relation to the hepatic vasculature. Hepatic veins are shown in blue, and portal vein are shown in red. LPV Left portal vein; MPV Main portal vein; RPV Right portal vein

of the data, yield images rivaling angiographic images obtained through more invasive techniques.

Three-dimensional applications and clinical examples: Precise localization of focal lesions is a common application of three-dimensional imaging in the liver. Surgical resection of colorectal metastases has become a viable treatment option, and three-dimensional imaging can help determine resectability and aid in procedure planning (10-12) (Figures $3,4)$. Angiographic reconstruction can be obtained following either intravenous injection of iodinated contrast or intra-arterial injection (ie, arterial portography). Three-dimensional imaging can be very useful in the preoperative evaluation of lesions situated near the portahepatis or the confluence of the subhepatic veins. Precise determination of portal vein, hepatic artery and biliary tree involvement is essential in planning resection of primary and metastatic tumours of the liver. Cholangiographic reconstructions can be obtained by exploiting the difference in appearance of the bile ducts compared with that of the surrounding, enhancing structures following intravascular administration of iodinated contrast. Although intravenous injection, three-dimensional processing may eventually replace angiography for certain pathologies, it can also be used in conjunction with intra-arterial, percutaneous and/or endoscopic administration of contrast to obtain volumerendered images.

Bile ducts - Spiral CT cholangiography: The rapid acquisition of large volumes of imaging information afforded by spiral imaging can be used for imaging patients with right upper quadrant pain. Thin slice volume acquisition of the biliary system after administration of intravenous cholangiographic contrast may offer a valuable alternative to patients who have difficult anatomy and, hence, are not candidates for endoscopic retrograde cholangiopancreatography (ERCP) and who cannot tolerate magnetic resonance cholangiography
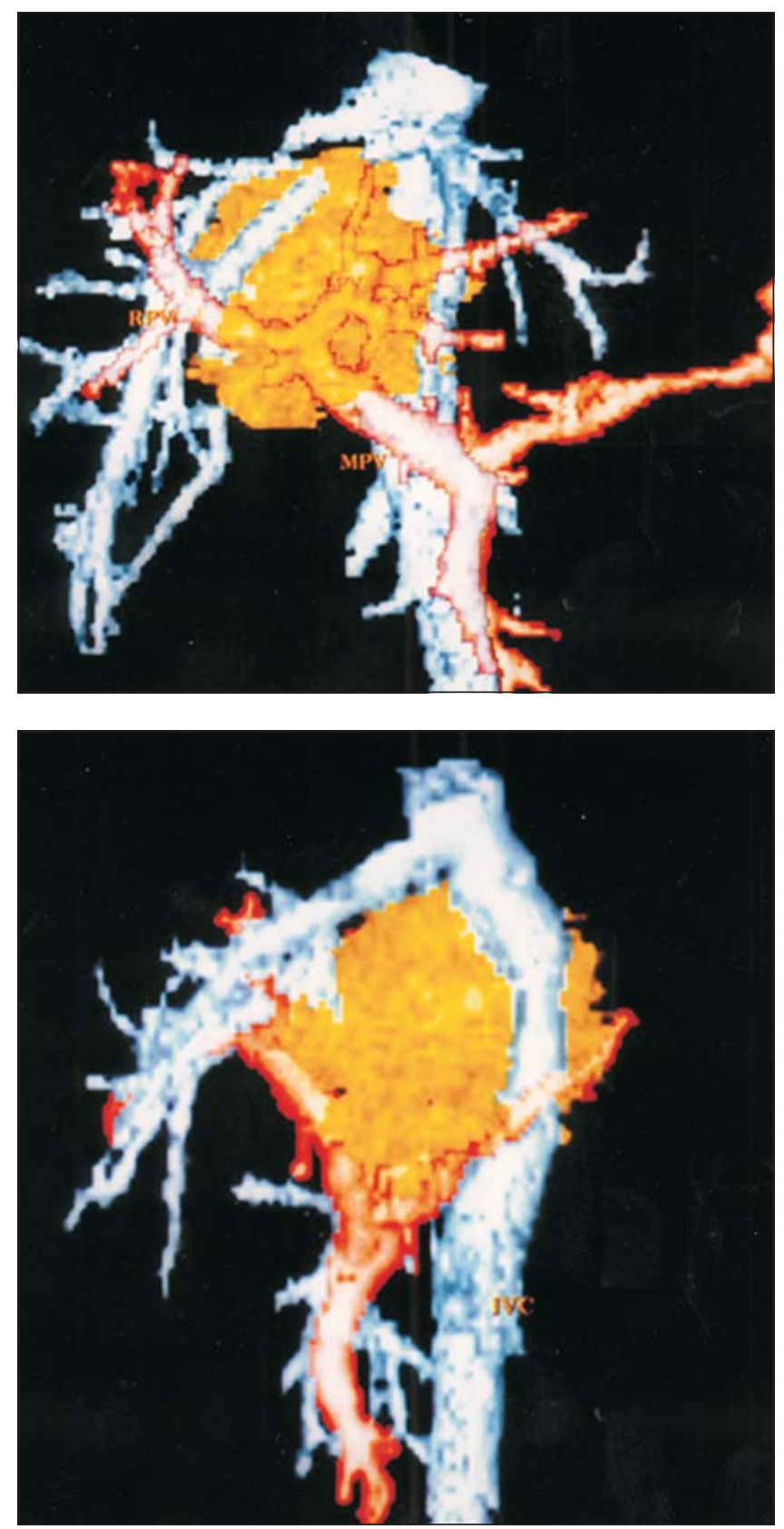

Figure 4) Spiral computed tomographic three-dimensional reconstructions of a malignant liver lesion (yellow) invading the inferior vena cava (IVC), as well as the portal vein bifurcation. Hepatic veins and IVC are shown in blue and portal veins are shown in red. LPV Left portal vein; MPV Main portal vein; RPV Right portal vein

(ie, claustrophobia). Several recent studies have documented the ability of spiral intravenous cholangiography to demonstrate clearly the normal anatomy as well as pathological entities such as cholelithiasis, choledocholithiasis and a variety of neoplastic entities in the pancreaticoduodenal region (13) (Figures 5,6). Addition of three-dimensional reconstruction allows pathological entities to be seen in an image format, which facilitates interpretation for the radiologist and the treating physician because these images closely resemble ERCP-generated studies. Again, the rapid 
acquisition of volume data allows the bile ducts to be studied adequately in one or two short (20 to $30 \mathrm{~s}$ ) breath-hold sequences.

More recently, unenhanced helical CT has been advocated for evaluating patients with suspected choledocholithiasis. In a study that used ERCP as the standard of reference, comprising 51 patients, unenhanced helical CT had a sensitivity of $88 \%$, a specificity of $97 \%$ and an accuracy of $94 \%$ in diagnosing common bile duct (CBD) stones (14) (Figures 7,8).

Pancreas - Pancreatic tumour staging: Pancreatic adenocarcinoma is an almost uniformly fatal disease; only a small minority of patients have resectable disease and, hence, hope for an extended survival. Preoperative staging of this neoplastic process is fraught with difficulty because of two major limiting factors - the inability to judge accurately tumour invasion or encasement of important vascular structures in the vicinity of the tumour and the sometimes subtle extension of tumour to surrounding peripancreatic structures. Classically, angiography has been touted as the 'gold standard' for vascular involvement but has proved to be of limited usefulness in cases of subtle invasion and particularly less helpful for assessment of portal vein involvement due to the sometimes suboptimal opacification of portal venous structures. Angiographic studies also add little useful information with regard to local tumour extension into surrounding areas, which can exclude patients from a curative resection.

Rapid acquisition CT spiral volume data in both arterial

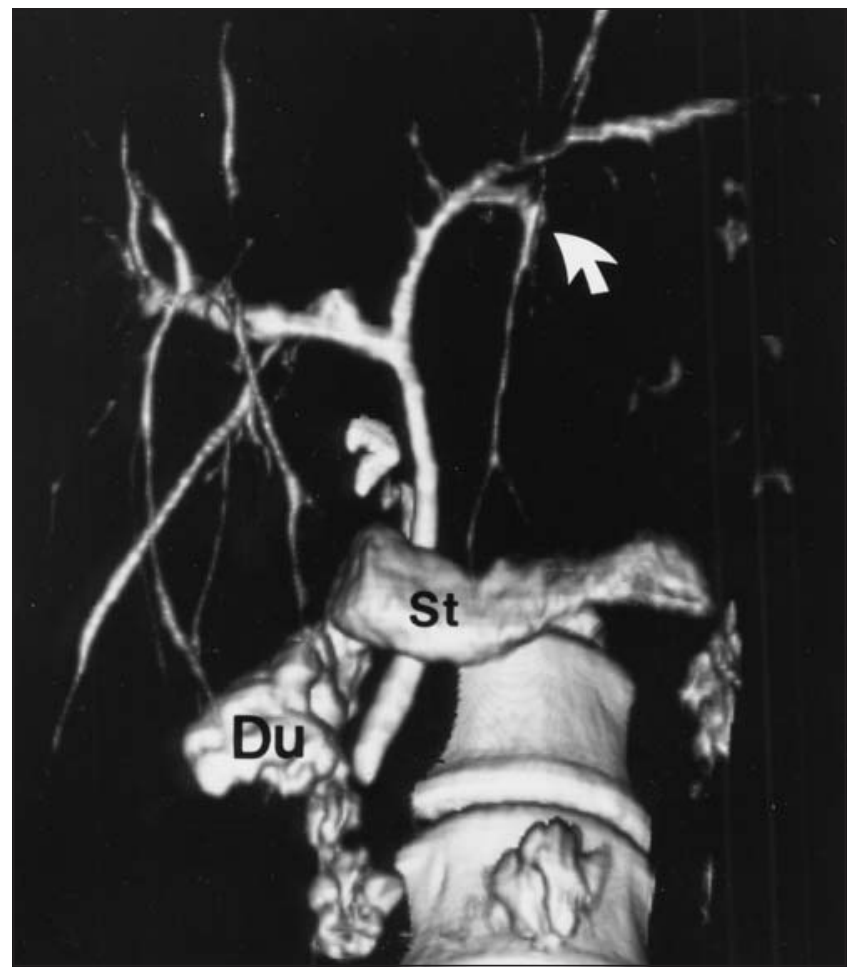

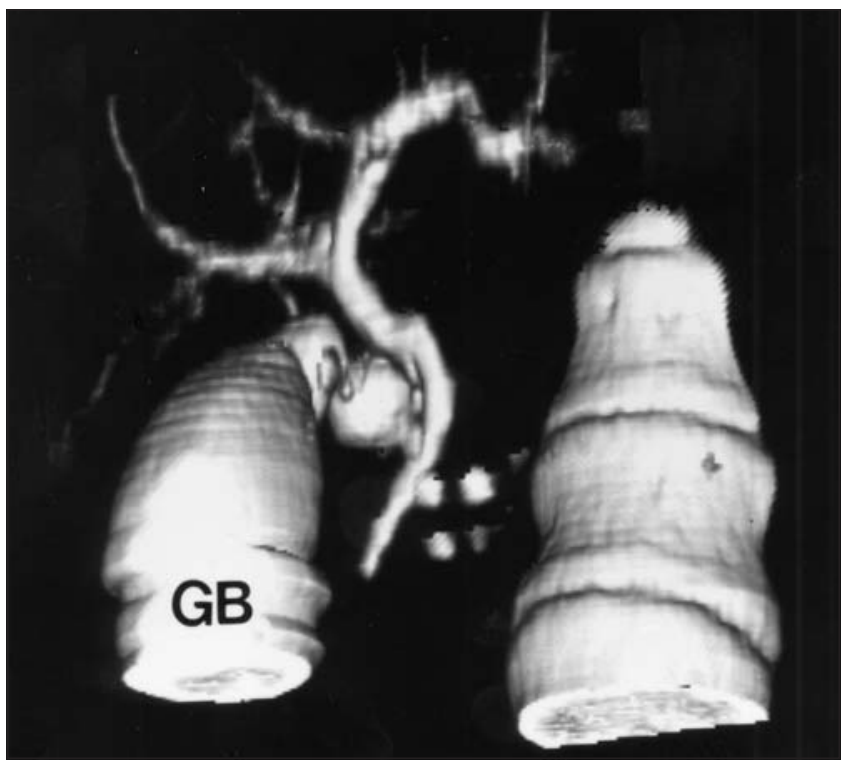

Figure 5) Spiral computed tomographic cholangiograph showing normal bile ducts. Three-dimensional surface reconstruction of a thin slice volume acquisition of the biliary tree demonstrates normal intra- and extrahepatic bile ducts. GB Gallbladder

and portal phases may offer a new 'gold standard' for tumour extension in these patients (15). Arterial acquisitions allow accurate assessment of any outright encasement or more subthe cases of vascular involvement by the tumour process. In addition, the arterial phase allows clear distinction between

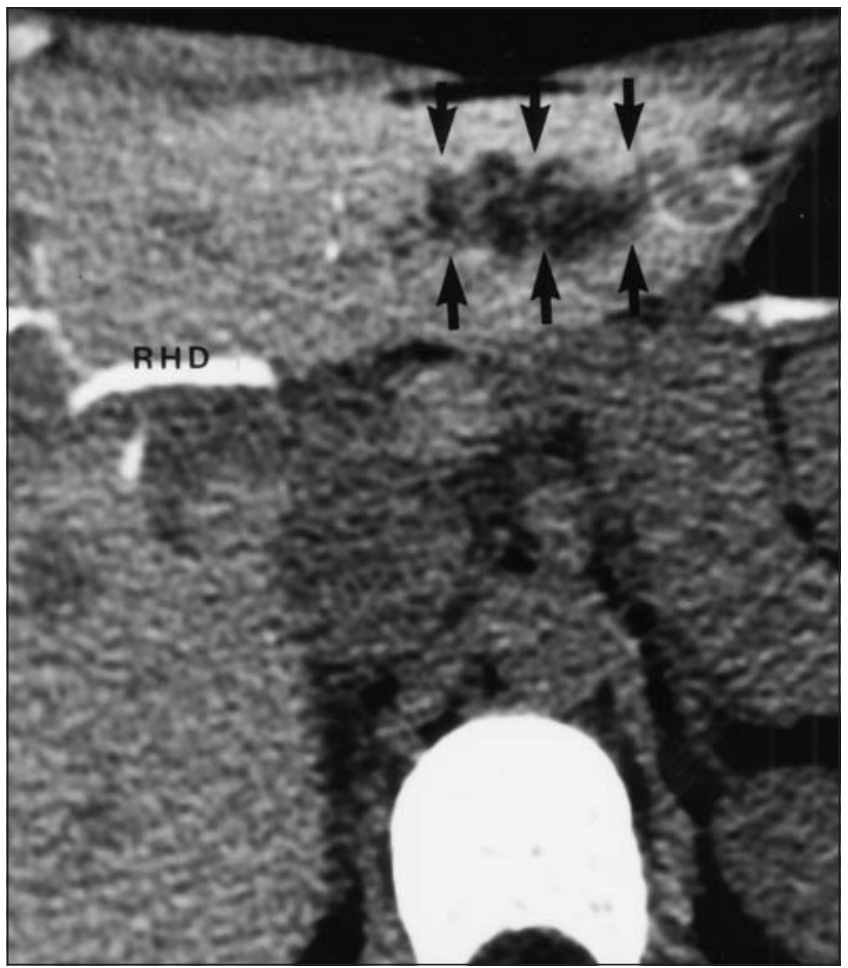

Figure 6) Spiral computed tomographic cholangiograph showing intrahepatic lithiasis. Left Three-dimensional surface reconstruction of a thin slice volume acquisition of the biliary tree. There is a cutoff of the duct-draining segment 3 of the liver (curved arrow). Right Axial source image. Unopacified bile ducts with multiple stones (arrows) are seen that are not visible on the three-dimensional cholangiogram (left). Du Duodenum; RHD Right hepatic duct; St Stomach 

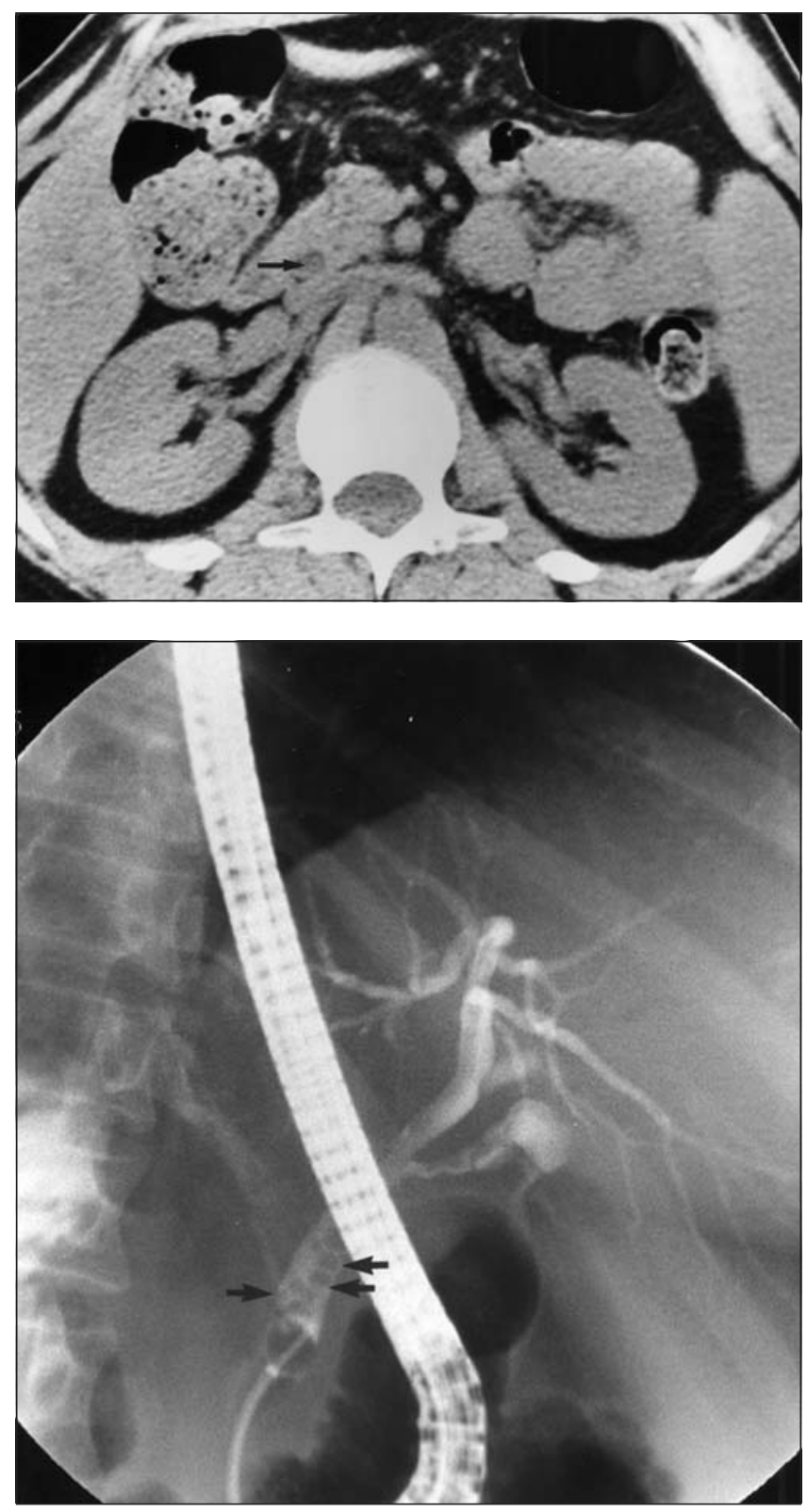

Figure 7) Unenhanced spiral computed tomograph. Top Unenhanced axial source image. There is a 'ring' of high density (arrow) in the distal common bile duct (CBD) consistent with a stone. Bottom Endoscopic retrograde pancreatograph confirms the presence of multiple stones (arrows) in the distal CBD. Photos courtesy of Dr Jeffrey D Neitlich, Yale University School of Medicine, New Haven, Conneticut

normal and pathological pancreatic tissue, and may demonstrate tumour extension in the peripancreatic tissues. A second acquisition in the portal venous phase allows accurate assessment of tumour involvement of the critical portal venous structures. In contradistinction to angiographic studies, adequate opacification of portal venous structures is easier to achieve with spiral CT studies because of the superior contrast sensitivity of computed tomography.

Added benefits of a two-phase, spiral CT acquisition include the assessment of metastatic disease to the liver, and evaluation of the presence of significant adenopathy or ma-
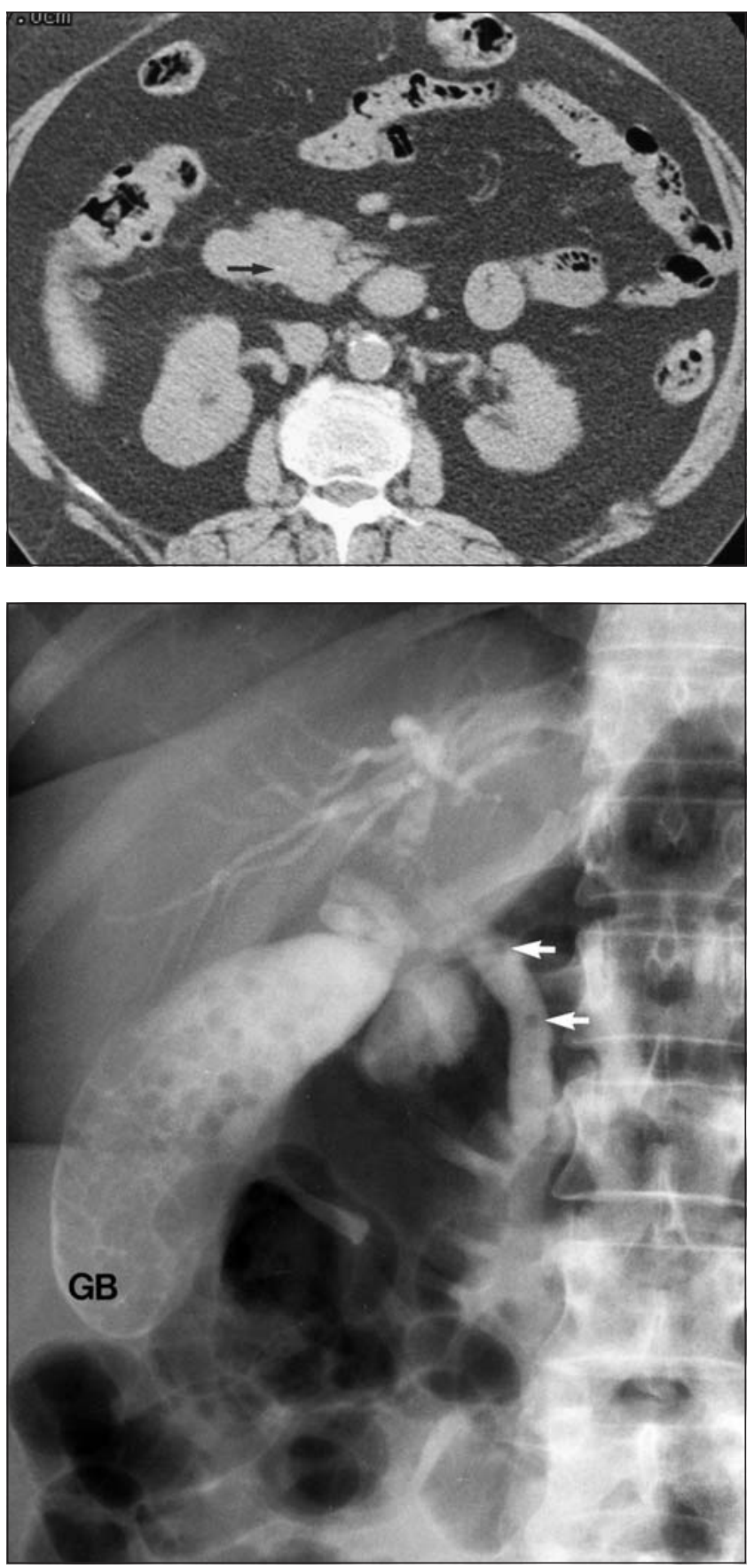

Figure 8) Unenhanced spiral computed tomograph. Top Unenhanced axial source image. There is a faint hyperdensity (arrow) in the head of the pancreas in the course of the distal common bile duct (CBD) consistent with a stone. Bottom Endoscopic retrograde cholangiopancreatography confirms the presence of multiple CBD stones (arrows). Note the presence of multiple gallstones. GB Gallbladder. Photos courtesy of Dr Jeffrey D Neitlich, Yale University School of Medicine, New Haven, Connecticut

lignant ascites. Reconstruction of the axial images into three-dimensional angiographic images offers another important benefit of spiral CT imaging.

Pancreatic three-dimensional imaging: In pancreatic disease, three-dimensional imaging is most useful in tumour staging and evaluating resectability. The ability to recon- 

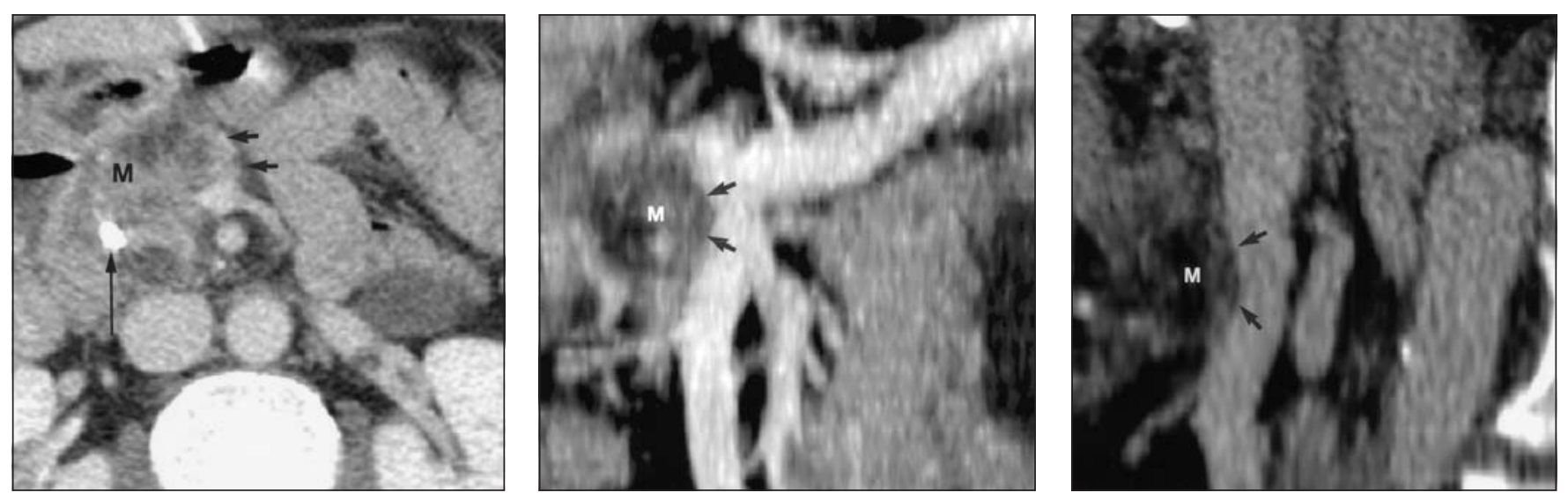

Figure 9) Spiral computed tomograph showing pancreatic adenocarcinoma staging. Left Axial source image. There is a hypodense mass (M) in the head of the pancreas, which partially surrounds the superior mesenteric vein (arrows). Incidentally, an endobiliary stent (long arrow) is situated within the common bile duct. Anterior (centre) and lateral (right) views of a thin slab volume reconstruction show the tumour mass (M) invading (arrows) the superior mesenteric vein to better advantage than the source images

struct angiographic projections of both the arterial and the portal systems is a valuable adjunct in evaluating vascular invasion (16-18) (Figure 9). The three-dimensional images compare well with traditional angiographic images and may allow patients to avoid more invasive procedures. Tumours of the periampullary region can invade the mesentericportal vein confluence. The degree of invasion is often difficult to assess on axial CT images as well as on traditional angiographic studies of the portal system. Three-dimensional reconstructions of the portal and superior mesenteric veins can display the vessel contours in exquisite detail and show subtle tumour invasion. Vascular mapping and identification of common anatomical variants can be easily accomplished by three-dimensional reconstruction of data obtained from spiral CT. Essentially, three-dimensional reconstructions improve the evaluation of the arterial and portal venous systems in the preoperative assessment of both benign and malignant pancreatic disease.

\section{MRI OF THE PANCREAS AND BILIARY TREE}

Introduction to MRI: Until recently, MRI has played only a minor role in the evaluation of patients with suspected pancreatic or biliary tract disease due to several technical limitations. However, recent advancements in imaging protocols have resulted in improved resolution (19), permitting evaluation of many neoplastic, inflammatory and congenital diseases of the pancreas and biliary system.

MRCP allows direct visualization of the biliary tree and pancreatic duct, similar to contrast cholangiography. The principle of MRCP is based on the acquisition of a heavily T2-weighted pulse sequence, which results in solid organs and moving fluids having a low signal intensity, whereas stationary fluids, including bile, have a relatively high signal intensity. It provides both high quality cross-sectional images of extraductal structures, and projectional (coronal) images of the biliary tree and pancreatic duct in a noninvasive examination that does require the administration of contrast material (20). It is in this respect that MRCP has the poten-

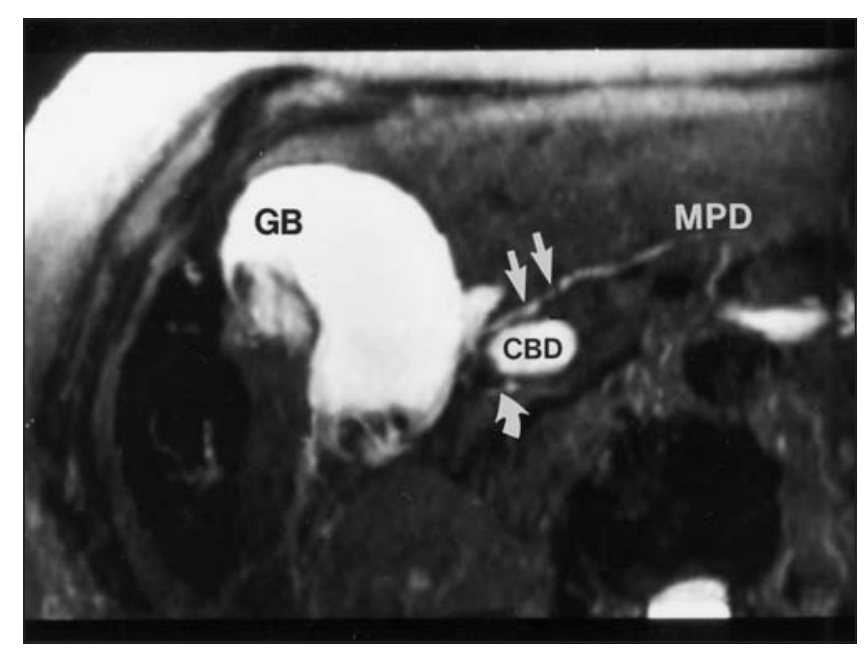

Figure 10) Magnetic resonance cholangiopancreatograph of pancreas divisum. Axial, targeted maximum intensity projection. The main pancreatic duct (MPD) is in direct continuity with the dorsal pancreatic duct (straight arrows), which courses anterior to the common bile duct (CBD). The ventral duct (curved arrow) shows no communication with the MPD. Incidentally noted are stones within the gallbladder (GB)

tial of replacing more traditional ways of imaging the pancreaticobiliary system, rather than adding to the already existing battery of available imaging tests.

Contrast agents such as gadolinium (gadolinium diethylene triamine penta-acetic acid [Gd-DTPA]) and manganese (manganese N,N'-bis-[pyridoxal 5-phosphate]ethylenediamine-N,N'-diacetic acid) chelates can be used for visualization of the intra- and extrahepatic bile ducts, gallbladder and pancreas with conventional MRI sequences $(21,22)$. Oral contrast agents may also be used to provide contrast between the duodenum and pancreas (19).

Normal anatomical variants: The accuracy of MRCP in diagnosing normal anatomical variants of the biliary tree has not yet been established. Taourel et al (23) detected anomalies, including low cystic duct insertion in $9 \%$ of patients, a medial cystic duct insertion in $17 \%$, a parallel course of the 

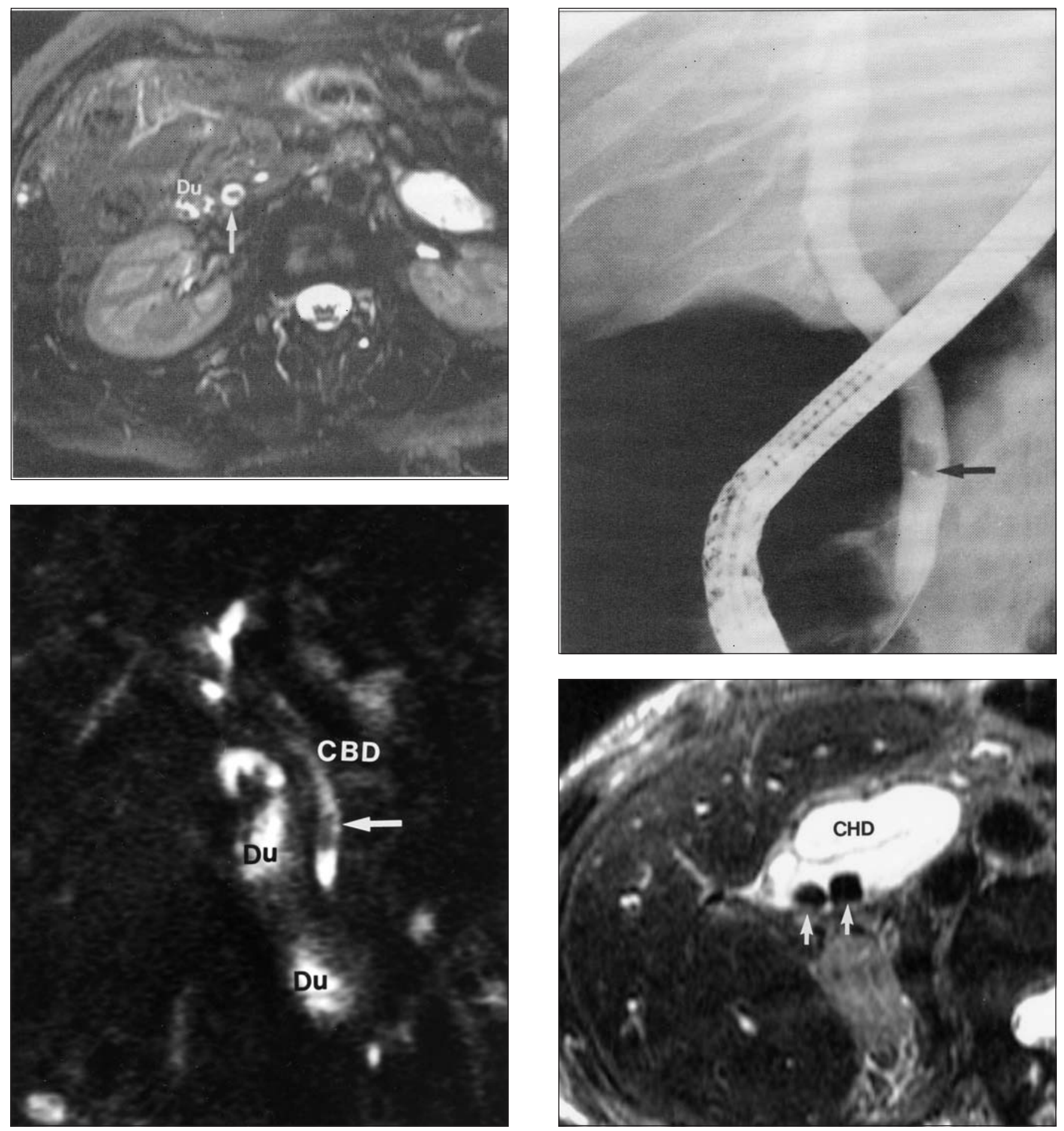

Figure 11) Magnetic resonance cholangiopancreatograph (MRCP) showing biliary lithiasis. Top left Axial MRCP source image. There is a low signal intensity area (arrow) in the distal common bile duct (CBD) consistent with a stone. Note that the CBD is mildly dilated. Bottom left Coronal MRCP source image in a different patient. There is a quadrangular area of low signal intensity (arrow) in a nondilated CBD. Top right Endoscopic retrograde cholangiopancreatograph in the same patient as shown in bottom left confirms the presence of a small quadrangular stone (arrow) in the distal CBD. Bottom right Axial MRCP source image. Two areas of signal void (arrows) consistent with stones are present in a moderately dilated cystic duct remnant. CHD Common hepatic duct; Du Duodenum

cystic and hepatic ducts in $25 \%$ and an aberrant right main hepatic duct in $9 \%$. This group of investigators found MRCP to be highly accurate in diagnosing these anatomical variants.

Pancreas divisum can be visualized on MRCP as a larger dominant dorsal pancreatic duct seen from the body to the head of the pancreas, crossing anteriorly to the CBD and draining separately and superiorly to the CBD (Figure 10). Visualization of the separate ventral duct draining into the major papilla is useful but not necessary for diagnosing pancreas divisum. The calibre of the ventral duct should be less 


\section{COPYRIGHT PULSUS GROUP INC, = DONOT COPY}

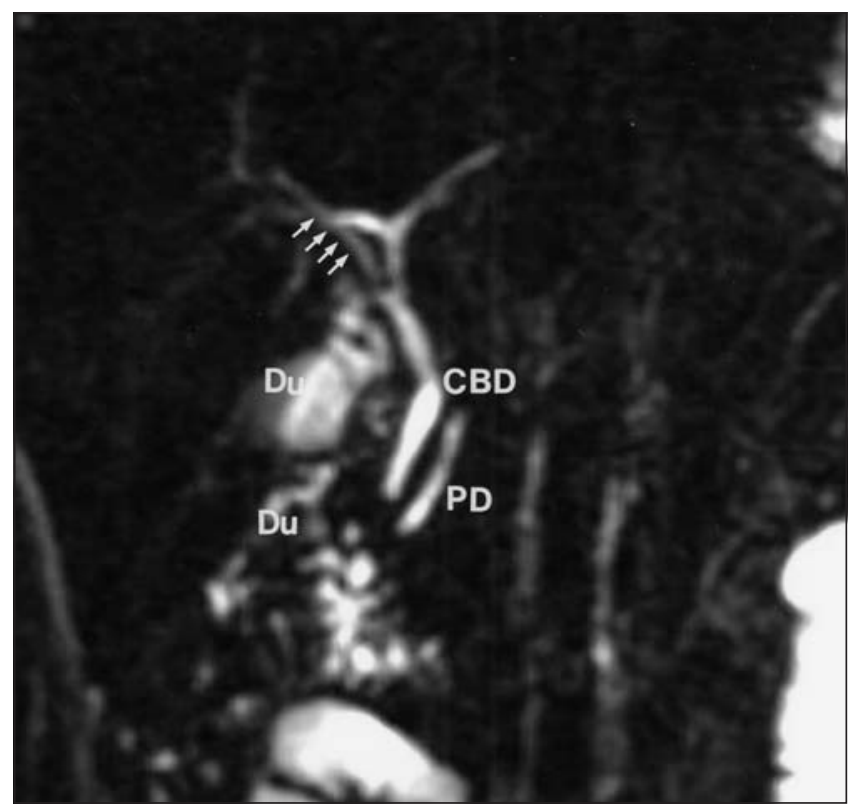

Figure 12) Magnetic resonance cholangiopancreatograph showing an aberrant right hepatic duct, with coronal maximum intensity projection. There is an aberrant right anterior hepatic duct (arrows) draining into the common hepatic duct. CBD Common bile duct; Du Duodenum; PD Pancreatic duct

than that of the dorsal duct. On ERCP, only the ventral duct is visualized from the standard major papilla cannulation, which results in incomplete ductography. The small ventral duct may be confused with another cause of main duct cutoff, such as malignancy. Diagnosing pancreas divisum by MRCP before ERCP may assist the endoscopist in preventing overinjection of the ventral duct, avoiding confusing a divisum for obstruction, and allowing cannulation of the minor papilla, as well obtaining a complete ductogram (24).

Until recently, pancreas divisum had only been reliably diagnosed by ERCP. In a series of 108 case studies imaged with MRCP where correlation with ERCP was available, all six cases of pancreas divisum were correctly identified, with no false positives or false negatives (25).

Other anomalies of the pancreaticobiliary junction include malunion of the pancreatic duct and CBD. MRCP was found to be effective in detecting anomalous pancreaticobiliary junctions in $82 \%$ of cases in a study comprising 159 patients (26). MRCP can also be useful in the diagnosis of rare anomalies such as annular pancreas, or partial or complete agenesis of the pancreas. MRCP combined with conventional MRI sequences has been reported to be as effective as ERCP in the detection of these anomalies $(27,28)$.

The biliary tree - Presence, location and cause of bile duct obstruction: Previous studies using MRCP to detect bile duct obstruction have demonstrated the presence of obstruction in $91 \%$ to $100 \%$ of cases $(29,30)$ and have been able to locate the level of obstruction in $91 \%$ to $100 \%(29,31)$. In addition, the bile duct distal to the site of obstruction was visualized in all patients in one study comprising 126 cases (29).
In distinguishing between a benign and malignant cause of bile duct obstruction, MRCP has been shown to have a sensitivity of $81 \%$ to $100 \%$ and a specificity of $92 \%$ to $100 \%$ (29-32). Guibaud et al (29) were able to characterize correctly the type of tumour in nine of 14 patients. This accuracy can be improved by the addition of contrast-enhanced conventional MRI sequences to the MRCP technique.

Biliary stones: Choledocholithiasis presents as an area of low signal within the bile ducts on MRCP, with a characteristic meniscus sign (Figure 11). However, this appearance is nonspecific, and other substances such as blood, proteinaceous debris, a polypoid mass or pneumobilia can mimic the presence of calculi (33). The differential diagnosis of pneumobilia and choledocholithiasis can be made by carefully observing axial MRIs in which air bubbles float anterior to bile and calculi lie in the dependent portion of the bile duct (34). However, this limitation also holds true for ERCP, where the differentiation of CBD stones from air bubbles is a common pitfall. To avoid the presence of pneumobilia, it is advisable that MRCP be performed before diagnostic ERCP or endoscopic sphincterotomy.

While ERCP remains the gold standard for diagnosing CBD stones, recent studies have examined the role of MRCP in the diagnosis of choledocholithiasis. MRCP diagnosed choledocholithiasis with a sensitivity of $81 \%$ to $92 \%$, a specificity of $91 \%$ to $98 \%$ and an overall diagnostic accuracy of $94 \%$ to $97 \%(29,31,32,35,36)$. MRCP may be useful when ultrasonography fails to detect stones despite a high clinical suspicion and to avoid ERCP unless necessary for therapeutic management. MRCP can also be useful after unsuccessful or incomplete ERCP (19), or in patients in whom there is an increased risk of complications from ERCP (33).

Bile duct injury: With the advent of laparoscopic cholecystectomy as the procedure of choice for patients with cholelithiasis, there has been an increase in the complication rate by up to $0.6 \%$ - as much as twice that of open cholecystectomy. The most common contributing factor to bile duct injury is aberrant bile duct anatomy - in particular aberrant right hepatic duct insertion in the common hepatic or cystic duct (up to $4 \%$ of patients) or medial insertion of the cystic duct into the CBD (12\% of patients) $(37,38)$ (Figure 12).

ERCP is the modality of choice to demonstrate bile leaks and bile duct injury. MRCP cannot demonstrate the actual leak but can only show a fluid collection in the gallbladder bed. MRCP most likely has little role in the diagnosis and management of simple leaks; however, for more complex leaks (eg, one or more excluded hepatic segments following complete transection of an aberrant right intrahepatic duct draining into the CBD), MRCP is the technique of choice. Surgical options depend on the distance between the proximal end of the lesion and the porta hepatis - information that is readily obtained by MRCP. When the injury involves one of the intrahepatic bile ducts, the unopacified bile duct segment can be overlooked with ERCP, whereas with MRCP, the presence of an excluded segment can be easily diagnosed and the associated dilation of the excluded bile 


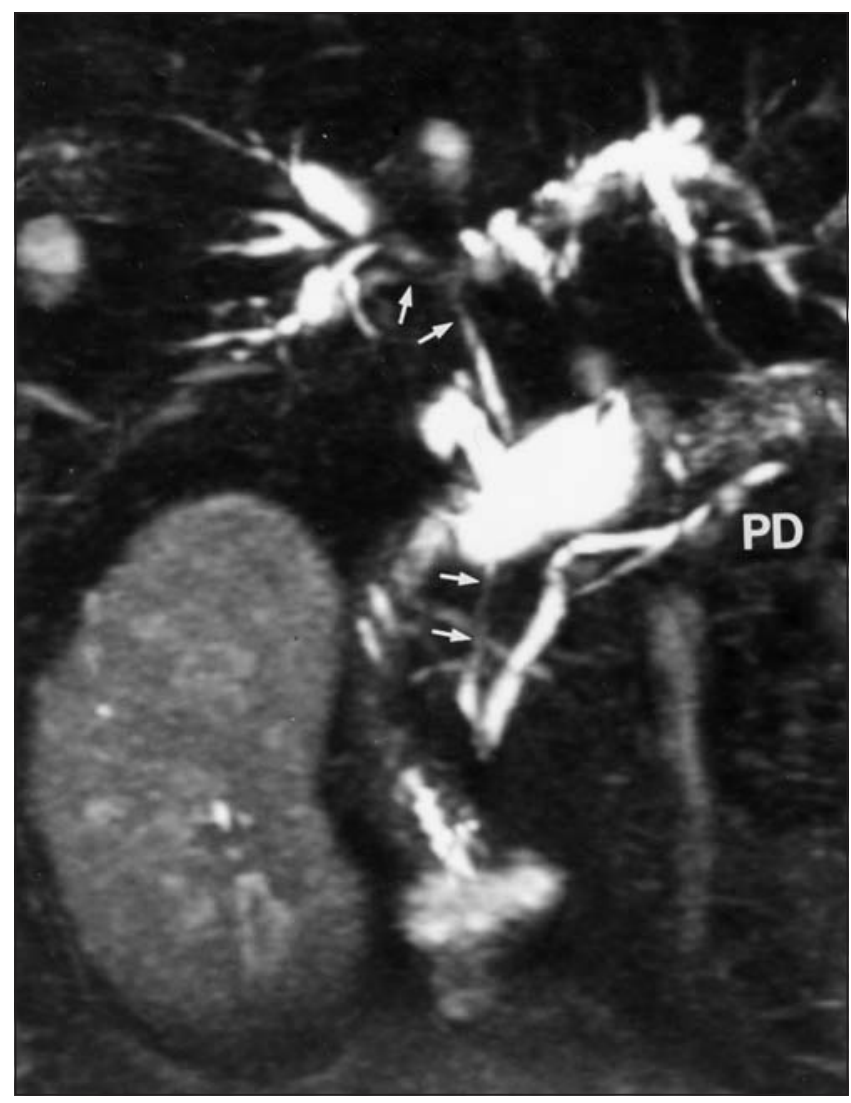

Figure 13) Magnetic resonance cholangiopancreatograph showing sclerosing cholangitis with coronal maximum intensity projection. Long strictures with smooth tapering ends (arrows) can be seen involving the common bile duct, as well as the common hepatic duct, with extension into the right and left main ducts. Note the beaded appearance of the intrahepatic bile ducts. PD Pancreatic duct

duct can be seen. MRCP and ERCP appear to be complementary modalities in the diagnosis and management of biliary complications of cholecystectomies, depending on the type of leak that is present (20).

Postoperative pancreaticobiliary anatomy: After a Whipple's procedure or roux-en-Y anastomosis, ERCP is technically very difficult and often cannot be performed successfully. In these cases, MRCP may be useful for visualization of the bile ducts and pancreatic duct proximal to the anastomosis (20). In a study comprising 35 patients who had previously undergone resection of the pancreas, MRCP was shown to be useful for follow-up screening tests in this patient group (39).

Sclerosing cholangitis: In patients with primary sclerosing cholangitis (PSC), once the large ducts are involved, the study of choice for establishing the diagnosis is cholangiography. A recent small study showed that PSC may have characteristic findings on MRCP that may have a role in the diagnosis of this disease (40) (Figure 13). Patients with PSC are at increased risk for developing cholangiocarcinoma and require regular follow-ups to monitor for signs of malignancy. In a small group of patients with PSC and known cholangiocarcinoma, a retrospective study showed definite or probable
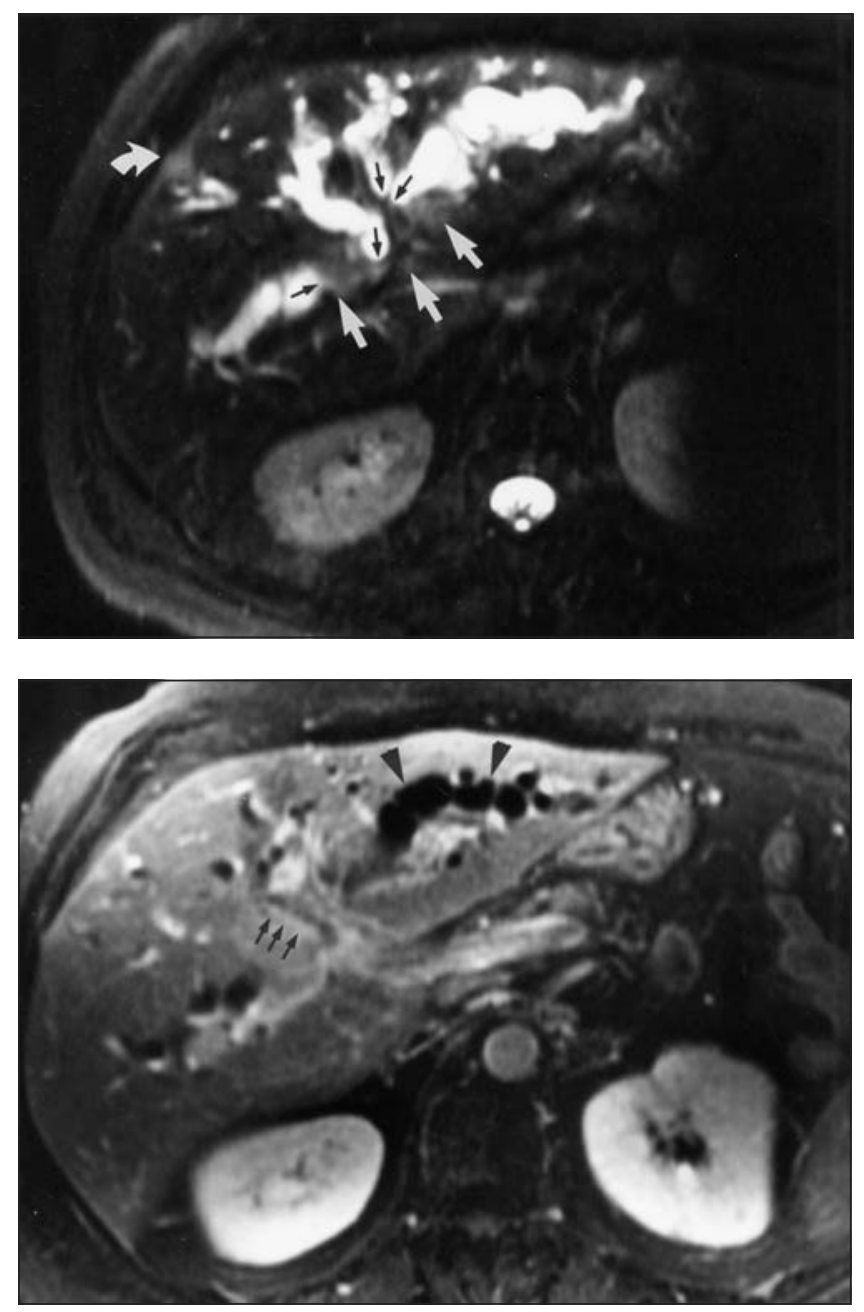

Figure 14) Magnetic resonance cholangiopancreatographic (MRCP) and conventional magnetic resonance images showing cholangiocarcinoma. Top Axial, targeted maximum intensity projection. Dilation of the left and right main bile ducts with segmental stenoses (small arrows) is shown on this MRCP image. A poorly defined mass of increased signal (arrows) is seen at the level of the obstruction. Note the presence of a small subserosal metastasis (curved arrow) of similar signal intensity. Bottom Gadolinium-enhanced T1-weighted spin-echo image shows to better advantage than MRCP the tumour spread along the bile ducts (small arrows). Compare this appearance with the uninvolved bile ducts whose walls (arrowheads) are almost imperceptible

cholangiocarcinoma in six of six patients studied with conventional MRI sequences (34).

Caroli's disease: Caroli's disease is characterized by saccular ectasia of the intrahepatic bile ducts and may involve the entire liver or be confined to a single segment. Several imaging modalities, including CT, ultrasonography and conventional MRI can demonstrate the bile duct cysts as fluidcontaining structures; however, direct cholangiography may be needed to show the communication between the cystic dilations and the biliary system and to define more accurately the type of cystic disease. In many patients, there is an increased risk of bacterial cholangitis with ERCP and percutaneous transhepatic cholangiography (PTC); however, MRCP can clearly demonstrate these bile-filled cystic dila- 


\section{COPYRIGHT PULSUS GROUP INC, = DONOT COPY}
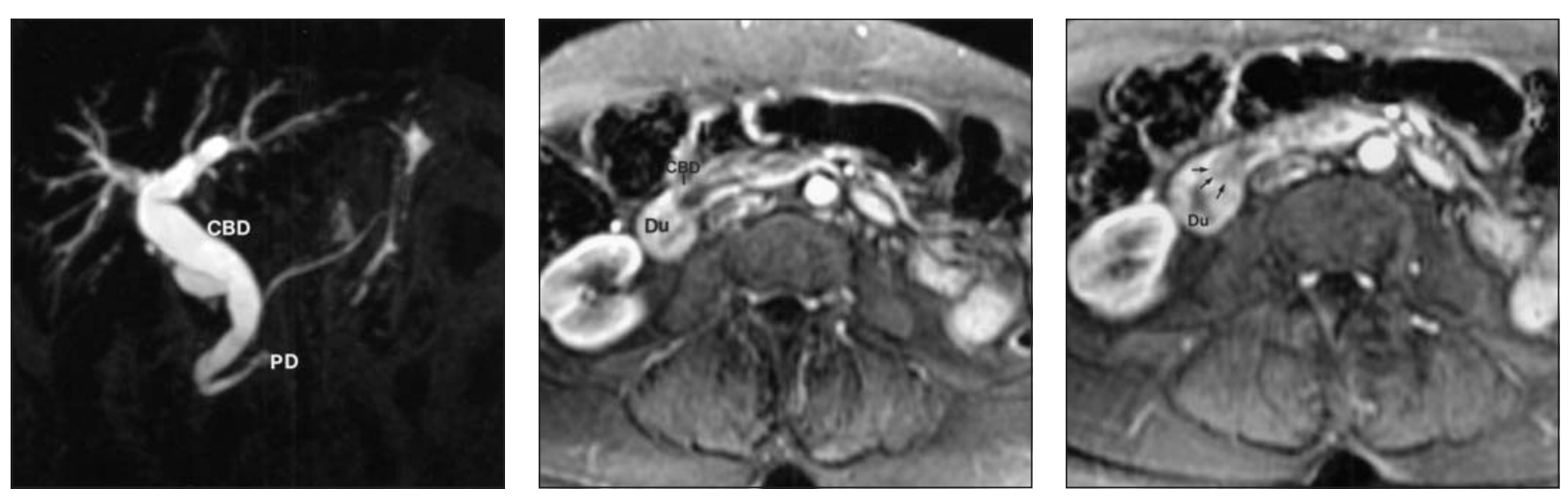

Figure 15) Magnetic resonance cholangiopancreatographic and conventional magnetic resonance images showing ampullary carcinoma. Left Coronal maximum intensity projection. There is moderate bile duct dilation to the level of the ampulla. Note minimal prominence of the distal pancreatic duct (PD) . Centre Axial contrast-enhanced conventional magnetic resonance image obtained at the level of the pancreatic head, showing a mildly dilated distal common bile duct (CBD) containing low signal intensity bile. Right A section taken $10 \mathrm{~mm}$ caudal to that shown in the middle image shows the presence of a small ampullary mass (arrows), which was proven at endoscopy to be an ampullary carcinoma. Du Duodenum

tions (41) and provide comparable images to direct cholangiogram in a few select cases that have been studied $(42,43)$. Cholangiocarcinoma: Although cholangiocarcinoma can usually be diagnosed using ultrasonography or CT, conventional MRI permits better detection and delineation of intrahepatic tumour extension than do contrast-enhanced CT images (44). The assessment of tumour extension along the bile ducts generally requires direct cholangiography. ERCP and PTC are limited in this application because ERCP cannot delineate the bile ducts proximal to the site of a total obstruction and PTC may incompletely map the bile ducts proximal to the lesion. The advantages of MRCP in this situation include its ability to provide a detailed map of the biliary tree anatomy and that it may provide more information than that available with ERCP or PTC. MRCP can also be used in the evaluation of hilar cholangiocarcinoma to determine the proximal extent of the disease, assessment of resectability and delineation of the duct both proximal and distal to the stricture and isolated bile duct obstructions (45). The coronal three-dimensional maximum intensity projection reconstructions simulate the images obtained on direct cholangiography and facilitate the identification of the various intrahepatic bile duct segments (Figure 14, top). The three-dimensional images provide information that is useful in planning surgery, palliative drainage and radiation therapy. As well, contrast-enhanced MRI sequences are suited for evaluation of tumour extension along the intrahepatic bile ducts (Figure 14, bottom) (20). Patients can be spared invasive procedures by the correlation of images of dilated ducts on MRCP and hepatic parenchyma on conventional MRI, which allows planning of optimal drainage by avoiding the drainage of atrophic segments. Complete staging, which includes involvement of liver, portal nodes or the portal vein, can be determined (20).

Ampullary carcinoma: Ampullary carcinomas, especially when intraductal, remain a challenge for MRCP. ERCP allows an endoscopic view of the ampulla and permits biopsy of any suspicious lesions. In a series of six surgically proven ampullary carcinomas diagnosed by MRCP from a group of 79 patients presenting with bile duct obstruction, only two cases of ampullary carcinoma were correctly diagnosed (Figure 15). Although future improvements in technique may permit better visualization of the ampulla by MRCP, the major papilla is currently visible in only $40 \%$ of patients on MRCP (20).

Pancreatic disease - Acute and chronic pancreatitis: In the diagnosis of acute pancreatitis, CT has been the imaging modality of choice to confirm the diagnosis and to look for potential complications used in planning the management of the patient (19). In a study that compared results in 32 patients with severe acute pancreatitis imaged with dynamic contrast-enhanced MRI and contrast-enhanced CT, it was found that MRI and CT were equivalent in distinguishing viable pancreatic tissue from areas of necrosis. While MRI appeared to be superior in characterizing the contents of fluid collections and in demonstrating gallstones, CT was more effective in detecting flecks of gas and calcification $(46,47)$.

Complications of acute pancreatitis such as pancreatic necrosis and acute hemorrhagic necrotizing pancreatitis can be imaged using contrast-enhanced MRI. While CT with contrast has been the imaging modality of choice for this diagnosis in the past, the iodinated contrast material puts these patients, who are often dehydrated, at increased risk for nephrotoxicity (48). As well, it has been shown that CT contrast material can increase the severity of necrotizing acute pancreatitis by aggravating further the impairment of pancreatic capillary blood flow. In animal studies, it has been demonstrated that the MRI contrast medium Gd-DTPA does not further impair pancreatic microcirculation or increase acinar injury in acute necrotizing pancreatitis (49).

Performing a diagnostic ERCP in the setting of acute gallstone pancreatitis remains controversial despite a randomized, clinical trial that demonstrated the benefit of performing an early ERCP and endoscopic sphincterotomy (50). However, complications with ERCP are encountered 

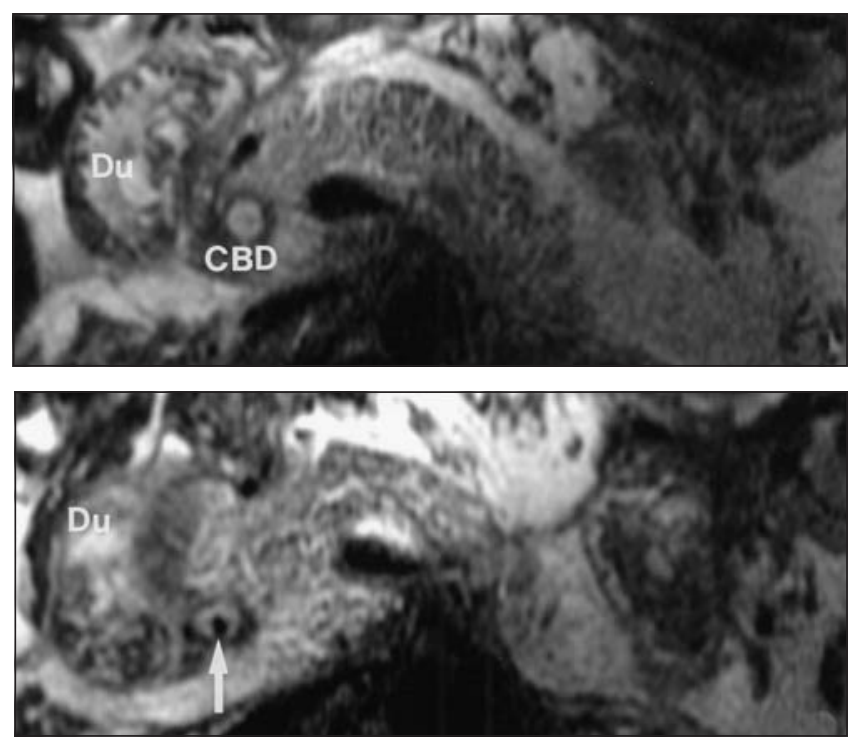

Figure 16) Magnetic resonance cholangiopancreatographic (MRCP) image showing acute pancreatitis. Axial MRCP source images. There is evidence of acute pancreatitis with peripancreatic fluid and pancreatic edema. Top An image obtained at the level of pancreatic head shows mild dilation of the common bile duct (CBD). Bottom A more caudal section shows the presence of a signal void (arrow) consistent with a stone in the distal CBD. Du Duodenum

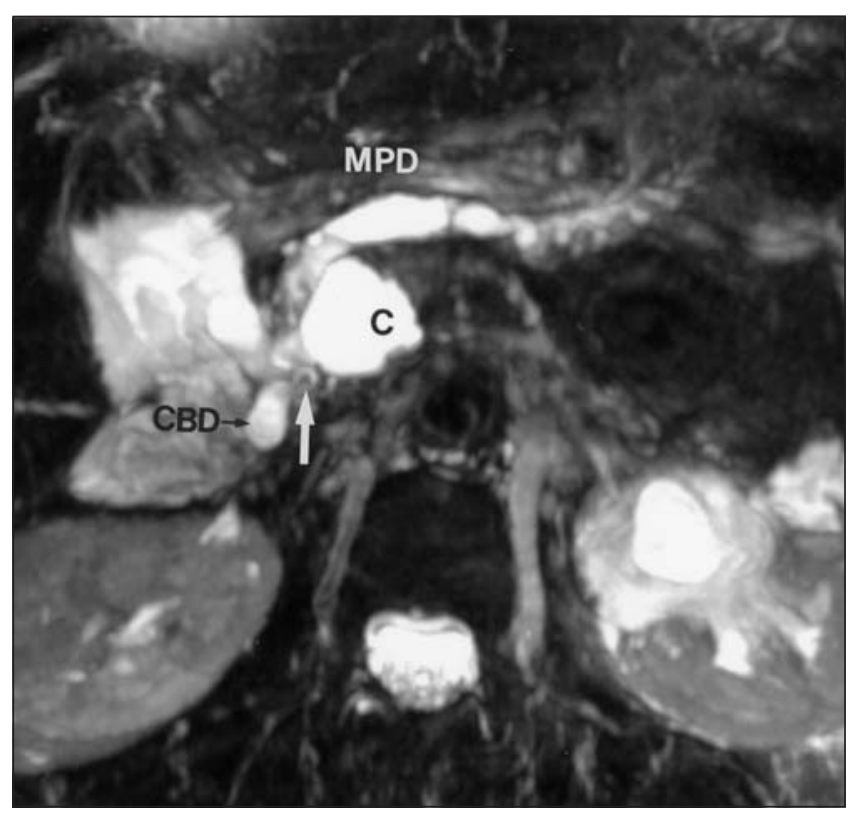

Figure 17) Magnetic resonance cholangiopancreatographic (MRCP) image showing chronic pancreatitis. Axial maximum intensity projection in a 78-year-old woman with chronic pancreatitis. The main pancreatic duct (MPD), as well as its side branches, are dilated. A filling defect (arrow) is present within the main pancreatic duct, consistent with a stone. A pseudocyst $(\mathrm{C})$ is present in the uncinate process of the pancreas. $\mathrm{CBD}$ Common bile duct

more frequently in patients with underlying pancreatitis (51). A more recent study showed no benefit of ERCP in patients unless they had continued jaundice or cholangitis (52). In this setting, MRCP may be particularly helpful, with positive cases referred for endoscopic stone removal and

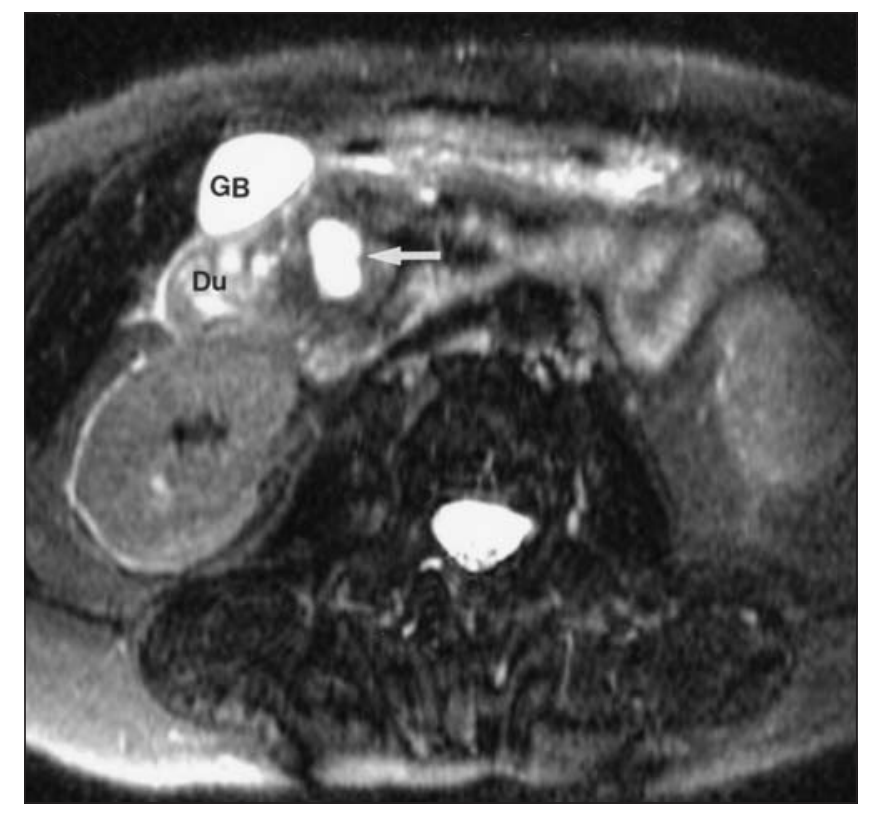

Figure 18) Magnetic resonance cholangiopancreatograph (MRCP) showing pancreatic pseudocyst. Axial MRCP source image in a 55year-old woman presenting with a pancreatic head mass and mild extrahepatic duct dilation. A unilocular cystic mass (arrow) consistent with a pseudocyst is present in the head of the pancreas. Two smaller pseudocysts are present in the duodenal wall. Du Duodenum; GB Gallbladder

negative cases with a low clinical suspicion for gallstone pancreatitis requiring no further investigation (Figure 16).

In chronic pancreatitis, MRI can demonstrate fibrosis and ductal abnormalities such as strictures, dilations and calculi. Intraluminal filling defects, which in the case of intraductal calculi are pathognomonic for chronic pancreatitis, can be accurately depicted with MRCP (53) (Figure 17). The sensitivity of MRCP in one study comprising $37 \mathrm{pa}-$ tients is $87 \%$ to $100 \%$ for duct dilation, $75 \%$ for ductal narrowing and $100 \%$ for ductal calculi (54). The administration of exogenous secretin before imaging with MRCP may be of benefit in the diagnosis of early disease in patients with chronic pancreatitis (55).

Once chronic pancreatitis has been diagnosed, the role of pancreatography is to assess disease progression, plan therapy and monitor progression. MRCP can readily be used to visualize the complications of chronic pancreatitis such as pseudocysts, intraductal obstruction by stones and bile duct dilation (Figures 17,18). Soto et al (56) used MRCP as the only pancreatographic technique in the successful surgical management of a small group of selected patients.

One of the critical issues in assessing pancreatic collections is to determine the best management for drainage. New techniques have allowed increasing numbers of percutaneous or endoscopic drainage, thus avoiding surgery in these patients. An important impediment to nonsurgical drainage, however, is the presence of residual necrotic debris in the fluid collection that significantly increases the risk of serious infection. In a study involving 18 patients, MRI correctly identified $100 \%$ of lesions that contained solid necrotic debris more than $1 \mathrm{~cm}$ in diameter and $100 \%$ of lesions that did 
not, which was superior to both ultrasonography $(88 \%$ and $54 \%$ ) and CT (25\% and $100 \%)$ (57).

Another potential use of MRI is in the differentiation of idiopathic from alcoholic chronic pancreatitis. In small groups of patients, autoimmune pancreatitis and nonalcoholic duct destructive chronic pancreatitis have been shown to have characteristic findings on MRI, which may be useful in the diagnosis of these entities $(58,59)$.

Pancreatic neoplasms: While CT has been the imaging modality of choice in routine clinical evaluations of pancreatic neoplasms, newer MRI sequences have been shown to be superior in both detecting and defining local tumour extension. Studies have found the sensitivity for tumour detection to be $69 \%$ to $74 \%$ for CT and $83 \%$ to $87 \%$ for MRI $(60,61)$. For tumours smaller than $3 \mathrm{~cm}$, MRI had a sensitivity of $67 \%$, and CT had a sensitivity of 53\% (60). One of the factors that correlates with patient outcome is tumour size; patients with small (smaller than $2 \mathrm{~cm}$ ) carcinomas can have up to a $30 \%$ five-year survival rate. One area where MRI may have an advantage over CT is in the detection of these small lesions (62) (Figure 19).

According to the report of the Radiology Diagnostic Oncology Group, published in 1995 (63), the accuracy for correctly assessing resectability of pancreatic cancer was found to be $73 \%$ for CT and $70 \%$ for MRI. This paper recommended the use of CT as the first choice in the imaging of pancreatic carcinoma. However, newer imaging sequences and the use of contrast agent administration in MRI have allowed conclusive diagnostic information when CT results were inconclusive (64). Ichikawa et al (65) retrospectively compared 21 patients in which both MRI and CT were used to evaluate tumour detection, local tumour extension and vascular involvement. A tumour was detected in $90 \%$ of MRI images and in $76 \%$ of helical CT scans. In a recent study, a combination of conventional MRI, MRI angiography (MRA) and MRCP in 23 patients with suspected pancreatic adenocarcinoma permitted correct assessment of resectability status in 22 of 23 patients (66). Similarly, a study using the same approach demonstrated MRI to be more accurate than other staging methods (ultrasonography, CT or angiography) in determining resectability (67). Other studies have found MRA to have a similar diagnostic value to that of the more invasive procedure of angiography (68). Advantages and limitations: A clear advantage of MRCP over ERCP is its lack of invasiveness. No adverse effects are encountered with MRI if patients are adequately screened for contraindications. In addition, routine sedation is not required with MRCP. MRCP is not operator dependent and can be easily implemented on most midfield or high field MRI scanners. The rate of failure of MRCP (due to contraindications and claustrophobia) is less than that of ERCP (31). In studies involving patients who had failed attempts at ERCP, for example due to altered anatomy after operation, follow-up MRCP provided diagnostic quality images in all patients $(56,69)$. MRCP allows visualization of the biliary tree and pancreatic duct both proximal and distal to the site of obstruction. This is particularly relevant in cases of multi-
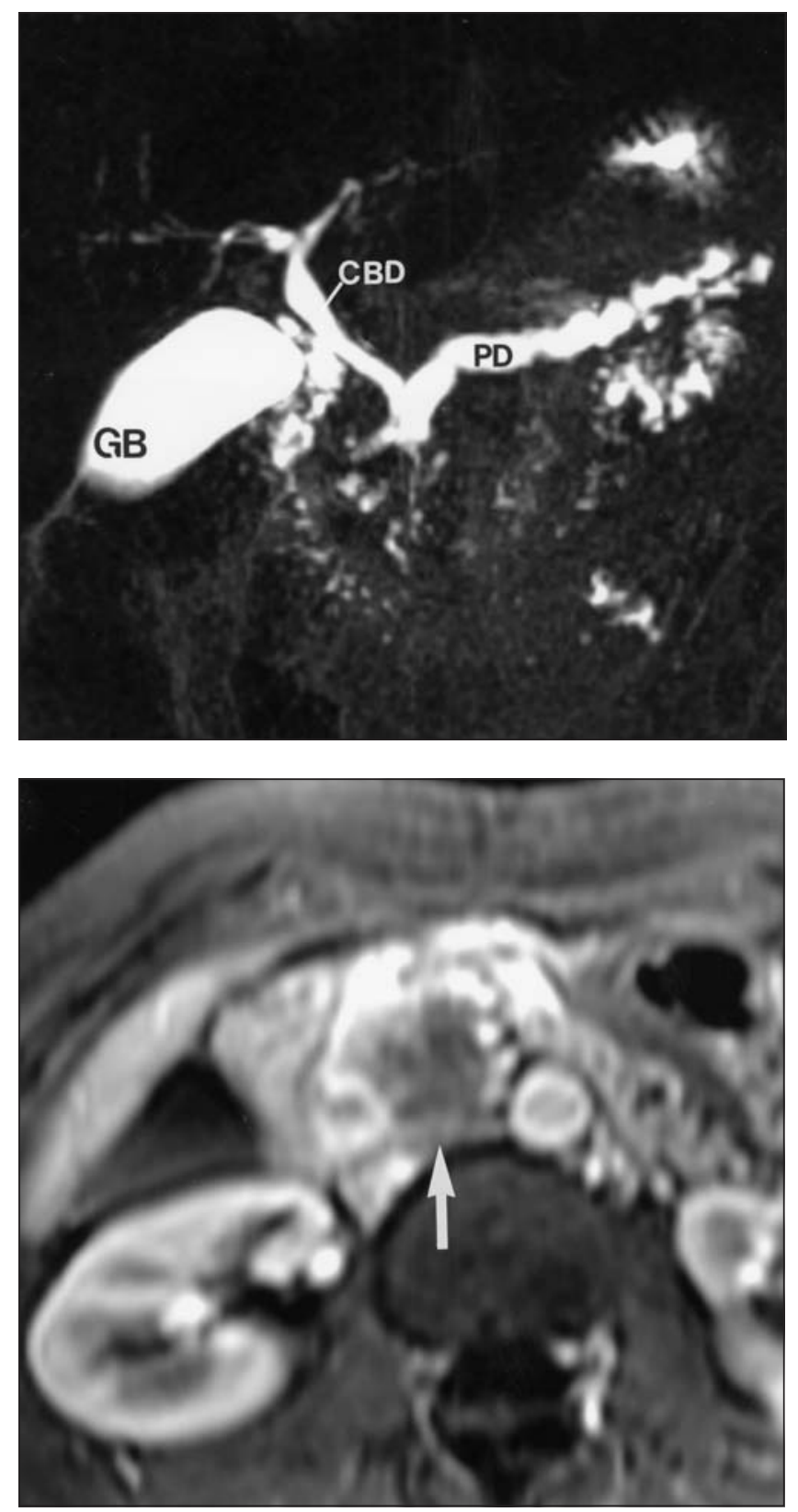

Figure 19) Magnetic resonance cholangiopancreatographic (MRCP) and conventional magnetic resonance images showing pancreatic adenocarcinoma. Top Coronal maximum intensity projection. There is dilation of the common bile duct (CBD) and pancreatic duct (PD), with a cutoff distally (reverse double duct sign). Bottom Gadolinium-enhanced T1-weighted gradient-echo image. There is a hypovascular mass (arrow) in the uncinate process of the pancreas proved to be an adenocarcinoma at surgery. Note the intense enhancement of the surrounding normal pancreatic parenchyma. GB Gallbladder

ple bile duct strictures, where a complete cholangiogram is needed to plan optimal therapeutic intervention. Image postprocessing, including multiplanar reformatting and three-dimensional reconstruction, allows MRCPs to be viewed in any desired imaging plane.

A current limitation of magnetic resonance cholangiography is the relatively low spatial resolution, which limits 
the visualization of nondilated pancreatic duct side branches, the characterization of strictures and the detection of CBD stones less than $4 \mathrm{~mm}$ in diameter (29). Signal loss from surgical clips after cholecystectomy may be seen with MRCP, although this rarely interferes with diagnostic accuracy. Finally, MRCP remains a diagnostic tool that cannot be used to provide therapeutic intervention, and most positive MRCP examinations require ERCP for definite treatment.

There are some contraindications to MRI, mostly related to the effect of the magnetic field on ferromagnetic objects. Absolute contraindications are cardiac pacemakers, due to the interference with pacemaker functioning in a strong external magnetic field and metallic ocular foreign bodies. In addition, certain models of cerebral aneurysm clips, ocular or cochlear implants, cardiac prosthetic valves, neurostimulators and penile implants are deflected by the magnetic field. A certain number of patients are not able to undergo MRI because of severe claustrophobia (20).

ERCP has several advantages, including the unparalleled resolution obtained with this technique and the ability to institute therapeutic measures at the time of initial diagnosis. However, the procedure is associated with a morbidity and mortality of $4 \%$ and $0.4 \%$, respectively, as reported in a recently published multicentre study (70). ERCP is also highly operator dependent, with unsuccessful cannulation of the CBD or pancreatic duct occurring in $5 \%$ of cases (70). Also, there is limited or no opacification of ducts proximal to a severe or complete obstruction, and the procedure requires routine sedation. ERCP is a relatively expensive technique when used for diagnostic purposes alone (71). A clear advantage of ERCP over MRCP is the ability to visualize the ampulla at endoscopy and to perform biopsies where indicated. Conclusions: MRCP is an important addition to more conventional MRI of the pancreaticobiliary system. It is too early to predict whether MRCP will be able to substitute for diagnostic ERCP routinely; however, it can clearly provide valuable information in patients for whom ERCP is unsuccessful or inadequate. To date, only preliminary results are available regarding the diagnostic accuracy of MRCP, and larger clinical trials are required. If the accuracy of MRCP approximates that of ERCP, cost effectiveness and outcome analysis studies will be needed to address the role of both techniques in the management of patients with suspected pancreaticobiliary disease. MRCP may be of use as a screening tool in determining which patient populations require invasive procedures such as ERCP or PTC for therapeutic means. It may also play a role in planning therapeutic drainage manoeuvres by providing more complete information on the ductal anatomy distal to the obstruction.

\section{SPIRAL CT COLONOGRAPHY FOR DETECTION OF COLORECTAL POLYPS}

Introduction to spiral CT colonography: Colorectal cancer is the second most common cause of cancer-related deaths in the United States (72). It is widely held that adenomatous polyps are the precursors of colorectal cancer and that re- moval of premalignant polyps decreases the incidence of colorectal cancer $(73,74)$. Barium enema and colonoscopy are two examinations that permit examination of the entire colon, with the highest accuracy rate obtained with colonoscopy. Although colonoscopy provides a means for both diagnostic screening and therapeutic intervention for removal of colonic polyps, it is invasive and the majority of patients with average risk have negative results (75).

Spiral CT colonography is a rapidly evolving technology that provides a noninvasive examination of the entire colon with a spectrum of two-dimensional and three-dimensional visualization techniques (76-84). With the continued advances and increasing availability of spiral CT, a dramatic improvement in the ability to acquire high resolution body CT scans has been achieved in routine clinical settings. These advances in data acquisition, coupled with improved computer graphics, have led to the interactive computersimulated visualization of the mucosal surface of the colon for polyp detection using spiral CT ('virtual colonoscopy'). Technical considerations: This spiral CT technique requires optimization of image acquisition, reconstruction and image processing for three-dimensional display. Image acquisition with spiral CT requires patients to undergo a routine bowel preparation before the scan. The patient is placed on the CT table, and air is insufflated per rectum. With single detector spiral CT scanners, a 30 to $40 \mathrm{~s}$ breath-hold acquisition of the abdomen and pelvis is acquired to image the entire colon. The images are typically acquired with $5 \mathrm{~mm}$ collimation and a pitch of 1.4 to 1.6 (table increment/collimation) - similar to routine abdomen protocols. Both prone and supine images are performed to achieve the best colonic distention of all colonic segments and decrease the impaired visualization from retained fluid. After CT acquisition, the patient is finished and can proceed directly to colonoscopy.

After image acquisition, image reconstruction of the data is performed to provide the resolution required for three-dimensional image display. Overlapping transaxial images are reconstructed at thin intervals of typically 1 to $2 \mathrm{~mm}$ compared with 5 to $7 \mathrm{~mm}$ in routine abdominal CT scans, thus generating a volumetric data set for high resolution two-dimensional and three-dimensional display. The volumetric data set is then transferred to a three-dimensional workstation for interactive viewing.

Multiple image display techniques are available to view the colon. Typically, two-dimensional multiplanar reformations (2D MPR) provide a time efficient survey of the entire colon, with simultaneous viewing of the axial, sagittal and coronal two-dimensional planes from an extraluminal orientation. The rapidly developing technique of threedimensional perspective volume rendering (3D PVR) applies multiple parameters, such as colour assignment and a diverging light source, to permit the endoscopic-like view of the colonic mucosal surface $(76,80)$. A field of view can be chosen in the 2D MPR, and the corresponding endoscopic 3D PVR view can be visualized interactively. Figures 1 and 2 illustrate the visualization of a small polyp and a cancer with the use of these two-dimensional and three-dimensional dis- 

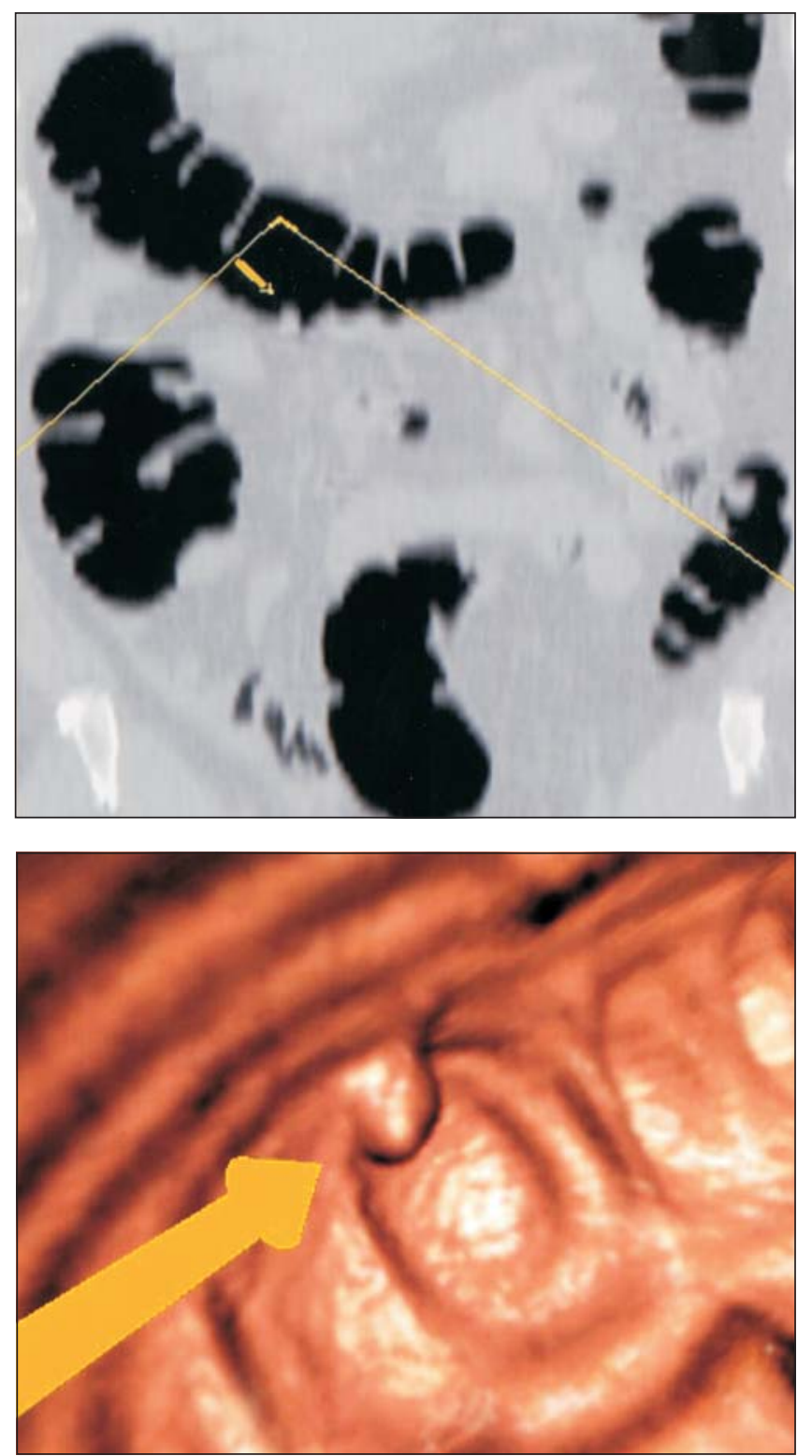

Figure 20) Computed tomographic colonoscopic image showing a colonic polyp. Top Coronal two-dimensional multiplanar reformatted image of an $8 \mathrm{~mm}$ polyp (yellow arrow) in the transverse colon. Bottom Corresponding intraluminal three-dimensional perspective volume image of the $8 \mathrm{~mm}$ polyp. Photos courtesy of Vitrea, Vital Images, Inc, USA

plays, respectively. Thus, different two-dimensional and three-dimensional displays allow seamless interaction of soft copy review to allow both intraluminal and extraluminal vantage points (Figures 20,21).

Accuracy of spiral CT colonography: To date, multiple institutions are investigating three-dimensional spiral CT techniques for detection of colorectal polyps. Published studies to date have established the feasibility of visualization of colonic polyps with a variety of both two-dimensional and three-dimensional techniques in phantom and limited patient investigations (76-86). To date, only a few prospective clinical studies have been published. In one series, 44 patients were evaluated with spiral
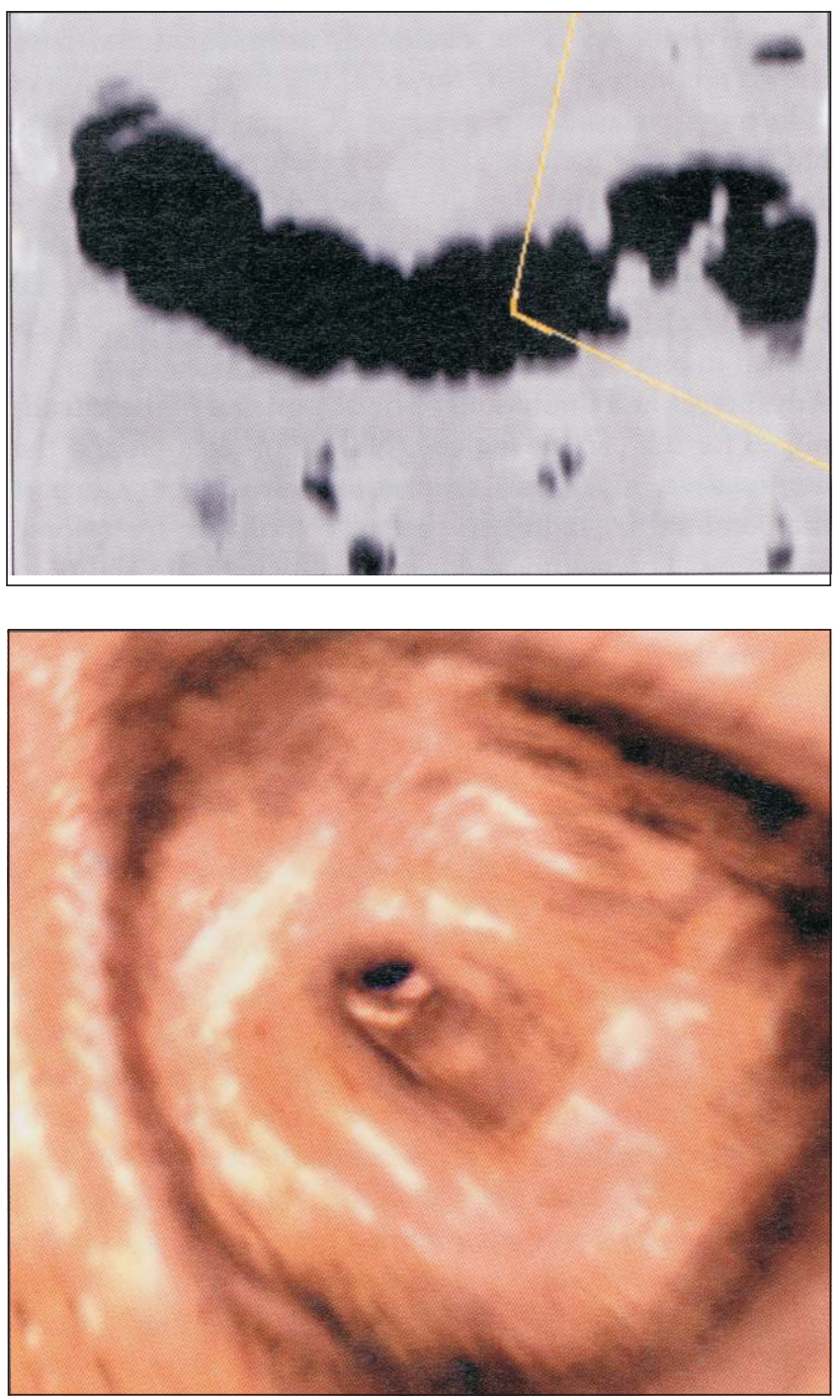

Figure 21) Computed tomographic colonoscopic image showing colonic carcinoma. Top Coronal two-dimensional multiplanar reformatted image of circumferential colon cancer in distal transverse colon, with retroflexed viewing angle. Bottom Corresponding intraluminal three-dimensional perspective volume-rendered image of colon cancer. Photos courtesy of Vitrea, Vital Images, Inc, USA

CT, with a total of 22 polyps confirmed at colonoscopy and evaluated independently by two radiologists (81). In this series, analysis by patient showed that the sensitivity ranged from $38 \%$ to $50 \%$ and that the specificity ranged from $86 \%$ to $93 \%$; analysis by polyp size showed that the sensitivity ranged from $83 \%$ for polyps $8 \mathrm{~mm}$ or greater $(\mathrm{n}=6), 33 \%$ for polyps 5 to $8 \mathrm{~mm}(\mathrm{n}=3)$ and $0 \%$ to $15 \%$ for polyps $5 \mathrm{~mm}$ or smaller $(n=13)(81)$. In another study of 70 patients with entrance criteria of either surveillance of prior polyps or known current polyps, 115 polyps were evaluated independently by two radiologists (78). In this series, analysis stratified by polyp size showed that the sensitivity ranged from $67 \%$ to $73 \%$ for polyps $10 \mathrm{~mm}$ or larger $(\mathrm{n}=15), 56 \%$ to $69 \%$ for polyps 5 to $9 \mathrm{~mm}(\mathrm{n}=21)$ and $25 \%$ to $27 \%$ for polyps smaller than $5 \mathrm{~mm}(\mathrm{n}=79)$. The positive predictive value (true posi- 
tive $\div$ true positive + false positive ratio) ranged from $35 \%$ in the intermediate polyps to $69 \%$ in the large polyps (78).

In addition to polyp detection, previous studies have shown preliminary results for spiral CT in the detection of colorectal cancers. In a series of 20 patients suspected to have colorectal cancer, 20 masses (defined as larger than $2 \mathrm{~cm}$ ) were correctly identified by spiral CT (79). Adequate insufflation of air beyond the mass was permitted, and complete CT examinations could be performed in 18 of the 20 patients, whereas a complete colonoscopy could be performed in only 12 patients.

Advantages and limitations: The advantages of these techniques for the patient include the lack of sedation and the noninvasive, rapid scan acquisition. These factors impart the potential for increased patient compliance for colonic screening. The technical advantages include the ability to reach the cecum in normal cases and to image the colon beyond an obstructing mass in the majority of cases. One of the most powerful imaging advantages is the fluid interactive visualization of the three-dimensional intraluminal viewpoint referenced with the corresponding extraluminal viewpoint in the 2D MPR. Anatomical orientation can be difficult for the endoscopist who is confined to an intraluminal view in a redundant colon. In addition, these techniques have the potential to quantify morphometric characteristics of the colon, such as length, diameter and wall thickness (82).

The disadvantages of three-dimensional imaging techniques in the past have been expense and lack of clinically practical protocols. However, recent advancements in computer technology have markedly driven down hardware costs of workstations, while maintaining equal or improved performance in rendering speed and spatial resolution. In the past, hardware requirements for a three-dimensional workstation ranged in excess of US\$150,000. Recently, however, workstations with similar capabilities are now available for less than US\$15,000. For colonic applications, technical difficulties include inadequate insufflation or retained fluid, which can prevent adequate visualization of some colonic segments. Prone and supine imaging, along with the use of smooth muscle relaxants, can help overcome these difficulties in most patients. Another limitation is the time needed to navigate through the colon in a continuous fashion. The automation of creating a central axis or through the lumen of the colon for the virtual examination is under investigation $(82,84,87)$. Spatial resolution of the mucosal surface detail is still a limitation. Although morphological contours of pedunculated polyps can be distinguished, the spatial resolution of the mucosal surface of flatter lesions is not possible. With improvements in CT acquisition, the ability to obtain subcentimeter slice thickness for improved spatial resolution is a future potential; however, radiation dose issues will have to be overcome.

Future technological advancements: There are several areas of rapid technological advancement in spiral CT colography. Stool tagging techniques are undergoing clinical trials that employ commercially available stool preparation kits
(E-Z-EM, Inc, USA). Patients ingest an oral sodium phosphate preparation along with an oral iodinated contrast agent before the CT examination, and then specialized CT stool substraction protocols are applied to the CT data to subtract the contrast-enhanced stool from the luminal contents of the colon (88). Further investigation is necessary to determine whether the conventional bowel preparation can be eliminated for improved patient compliance. Computer-aided diagnosis techniques are being explored to aid the radiologist in the detection of focal wall abnormalities (84). Continued advancements in the three-dimensional image display techniques have recently introduced digitally flattened views (89) and panoramic views (90), which ease the field of view restraints when confined to the intraluminal perspective. Further automation of real time auto navigation through the three-dimensional endoscopic mode are also being developed $(82,84,87)$.

The future of these techniques for the examination of the colon and other anatomical regions requires close collaboration among radiologists, computer scientists and the subspecialty referral physicians. Prospective trials comparing diagnostic performance between CT and endoscopy are needed to determine clinical utility. If proven accurate for the detection of colonic polyps, spiral CT colonography has the potential to improve patient compliance for colon cancer screening or surveillance.

\section{REFERENCES}

1. Bonaldi VM, Garcia P, Coche EE, Sarazin L, Bret PM. Spiral $\mathrm{x}$-ray computed tomography: a new modality of scanner imaging. Presse Med 1996;25:109-14.

2. Zeman RK, Baron RL, Jeffrey RB Jr, Klein J, Siegel MJ, Silverman PM. Helical body CT: Evolution of scanning protocols. AJR Am J Roentgenol 1998;170:1427-38.

3. Vannucchi L, Zanfranceschi G, Simonelli P, Niccolai F, Piperno G. Secondary liver lesions studied with double-helix spiral computed tomography: first experience. Radiol Med 1996;91:769-73.

4. Choi BI, Han JK, Cho JM, et al. Characterization of focal hepatic tumors. Value of two-phase scanning with spiral computed tomography. Cancer 1995;76:2434-42.

5. Miller FH, Butler RS, Hoff FL, Fitzgerald SW, Nemcek AA Jr, Gore RM. Using triphasic helical CT to detect focal hepatic lesions in patients with neoplasms. AJR Am J Roentgenol 1998;171:643-9.

6. Lupetin AR, Cammisa BA, Beckman I, et al. Spiral CT during arterial portography. Radiographics 1996;16:723-43.

7. Woodhouse CE, Ney DR, Sitzmann JV, Fishman EK. Spiral computed tomography arterial portography with three-dimensional volumetric rendering for oncologic surgery planning. A retrospective analysis. Invest Radiol 1994:29:1031-7.

8. Johnson PT, Heath DG, Kuszyk BS, Fishman EK. CT angiography with volume rendering: advantages and applications in splanchnic vascular imaging. Radiology 1996;200:564-8.

9. Kanematsu M, Imaeda T, Mizuno S, et al. Value of threedimensional spiral CT hepatic angiography. AJR Am J Roentgenol 1996;166:585-91.

10. Winter TC III, Freeny PC, Nghiem HV, et al. Hepatic arterial anatomy in transplantation candidates: evaluation with three-dimensional CT arteriography. Radiology 1995;195:363-70.

11. Togo S, Shimada H, Kanemura E, et al. Usefulness of three-dimensional computed tomography for anatomic liver resection: sub-subsegmentectomy. Surgery 1998;123:73-8.

12. Yoshimi F, Hasegawa H, Amemiya R, Koizumi S, Kobayashi H, Matsueda K. Application of three-dimensional spiral computed tomographic angiography prior to hepatectomy for hepatocellular carcinoma. Surg Today 1995;25:37-42.

13. Kwon AH, Uetsuji S, Yamada O, Inoue T, Kamiyama Y, Boku T. 
Three-dimensional reconstruction of the biliary tract using spiral computed tomography. Br J Surg 1995;82:260-3.

14. Neitlich JD, Topazian M, Smith RC, Gupta A, Burrell MI,

Rosenfield AT. Detection of choledocholithiasis: comparison of unenhanced helical CT and endoscopic retrograde

cholangiopancreatography. Radiology 1997;203:753-7.

15. Cunningham JD, Glajchen N, Brower ST. The use of spiral computed tomography in the evaluation of vessel encasement for pancreatic cancer. Int J Pancreatol 1996;19:9-14

16. Blomley MJ, Albrecht T, Williamson RC, Allison DJ.

Three-dimensional spiral CT angiography in pancreatic surgical planning using non-tailored protocols: comparison with conventional angiography. Br J Radiol 1998;71:268-75.

17. Novick SL, Fishman EK. Three-dimensional CT angiography of pancreatic carcinoma: role in staging extent of disease. AJR Am J Roentgenol 1998;170:139-43.

18. Raptopoulos V, Steer ML, Sheiman RG, Vrachliotis TG, Gougoutas CA, Movson JS. The use of helical CT and CT angiography to predict vascular involvement from pancreatic cancer: correlation with findings at surgery. AJR Am J Roentgenol 1997;168:971-7.

19. Mammone JF, Siegelman ES, Outwater EK. Magnetic resonance imaging of the pancreas and biliary tree. Semin Ultrasound CT MR 1998;19:35-52.

20. Mehta SN, Reinhold C, Barkun AN. Magnetic resonance cholangiopancreatography. Gastrointest Endosc Clin North Am 1997;7:247-70.

21. Bollow M, Taupitz M, Hamm B, Staks T, Wolf KJ, Weinmann HJ. Gadolinium-ethoxybenzyl-DTPA as a hepatobiliary contrast agent for use in MR cholangiography: results of an in vivo phase-I clinical evaluation. Eur Radiol 1997;7:126-32.

22. Kettritz U, Warshauer DM, Brown ED, Schlund JF, Eisenberg LB, Semelka RC. Enhancement of the normal pancreas: comparison of manganese-DPDP and gadolinium chelate. Eur Radiol 1996;6:14-8.

23. Taourel P, Bret PM, Reinhold C, Barkun AN, Atri M. Anatomic variants of the biliary tree: diagnosis with MR cholangiopancreatography. Radiology 1996;199:521-7.

24. Martin RF, Rossi RL. Bile duct injuries: spectrum, mechanisms of injury, and their prevention. Surg Clin North Am 1994;74:781-803.

25. Bret PM, Reinhold C, Taourel P, Guibaud L, Atri M, Barkun AN. Pancreas divisum: evaluation with MR cholangiopancreatography. Radiology 1996;199:99-103.

26. Sugiyama M, Baba M, Atomi Y, Hanaoka H, Mizutani Y, Hachiya J. Diagnosis of anomalous pancreaticobiliary junction: value of magnetic resonance cholangiopancreatography. Surgery 1998;123:391-7.

27. Lecesne R, Stein L, Reinhold C, Bret PM. MR

cholangiopancreatography of annular pancreas. J Comput Assist Tomogr 1998;22:85-6.

28. Macari M, Giovanniello G, Blair L, Krinsky G. Diagnosis of agenesis of the dorsal pancreas with MR pancreatography. AJR Am J Roentgenol 1998;170:144-6.

29. Guibaud L, Bret PM, Reinhold C, Atri M, Barkun AN. Bile duct obstruction and choledocholithiasis: Diagnosis with MR cholangiography. Radiology 1995;197:109-15.

30. Bearcroft W, Gimson A, Lomas DJ. Non-invasive cholangio-pancreatography by breath-hold magnetic resonance imaging: preliminary results. Clin Radiol 1997;52:345-50.

31. Lee MG, Lee HJ, Kim MH, et al. Extrahepatic biliary diseases: 3D MR cholangiopancreatography compared to endoscopic retrograde cholangiopancreatography. Radiology 1997;202:663-9.

32. Lomanto D, Pavone P, Laghi A, et al. Magnetic resonance cholangiopancreatography in the diagnosis of biliopancreatic diseases. Am J Surg 1997;174:33-8.

33. Barish MA, Soto JA. MR cholangiopancreatography: techniques and clinical applications. AJR Am J Roentgenol 1997;169:1295-303.

34. Campbell WL, Ferris JV, Holbert BL, Thaete FL, Baron RL. Biliary tract carcinoma complicating primary sclerosing cholangitis: evaluation with CT, cholangiography, US and MR imaging. Radiology 1998;207:41-50.

35. Becker CD, Grossholz M, Becker M, Mentha G, de Peyer R, Terrier F. Choledocholithiasis and bile duct stenosis: diagnostic accuracy of MR cholangiopancreatography. Radiology 1997;205:523-30.

36. Reinhold C, Taourel P, Bret PM, et al. Choledocholithiasis: Evaluation of MR cholangiography for diagnosis. Radiology 1998;209:435-42.
37. Traverso LW, Hauptmann EM, Lynge DC. Routine intraoperative cholangiography and its contributions to the selective cholangiographer. Am J Surg 1994;167:464-8.

38. Lehman GA, Sherman S. Pancreas divisum: diagnosis, clinical significance and management alternatives. Gastrointest Endosc Clin North Am 1995;5:145-70.

39. Ishizu H, Takahashi M, Kondo Y, et al. The role of magnetic resonance cholangiopancreatography (MRCP) after resection of the pancreas. Surg Today 1997;27:285-7.

40. Ernst O, Asselah T, Sergent G, et al. MR cholangiopancreatography in primary sclerosing cholangitis. AJR Am J Roentgenol 1998;171:1027-30.

41. Vrachiotis TG, Shirkhoda A, Bis KG, et al. MR cholangiopancreatography (MRCP). Crit Rev Diagn Imaging 1997;36:295-323.

42. Asselah T, Ernst O, Sergent G, L'hermine C, Paris JC. Caroli's disease: a magnetic resonance cholangiopancreatography diagnosis. Am J Gastroenterol 1998;93:109-10.

43. Pavone P, Laghi A, Catalano C, Materia A, Basso N, Passariello R. Caroli's disease: evaluation with MR cholangiopancreatography (MRCP). Abdom Imaging 1996;21:117-9.

44. Choi BI, Han JK, Shin YM, Baek SY, Han MC. Peripheral cholangiocarcinoma: comparison of MRI with CT. Abdom Imaging 1995;20:357-60.

45. Fulcher AS, Turner MA. HASTE MR cholangiography in the evaluation of hilar cholangiocarcinoma. AJR Am J Roentgenol 1997;169:1501-5

46. Ward J, Chalmers AG, Guthrie AJ, Larvin M, Robinson PJ. T2-weighted and dynamic enhanced MRI in acute pancreatitis: comparison with contrast enhanced CT. Clin Radiol 1997;52:109-14.

47. Lecesne R, Taourel P, Bret PM, Atri M, Reinhold C. Acute pancreatitis: interobserver agreement and correlation of CT and MR cholangiopancreatography with outcome. Radiology 1999;211:727-35.

48. Piironen A, Kivisaari R, Pitkaranta P, et al. Contrast-enhanced magnetic resonance imaging for the detection of acute haemorrhagic necrotizing pancreatitis. Eur Radiol 1997;7:17-20.

49. Werner J, Schmidt J, Warshaw AL, Gebhard MM, Herfarth C, Klar E. The relative safety of MRI contrast agent in acute necrotizing pancreatitis. Ann Surg 1998;227:105-11.

50. Fan ST, Lai EC, Mok FP, Lo CM, Zheng SS, Wong J. Early treatment of acute biliary pancreatitis by endoscopic papillotomy. N Engl J Med 1993;328:228-32.

51. Sherman S, Lehman GA. ERCP and endoscopic sphincterotomy induced pancreatitis. Pancreas 1991;6:350-67.

52. Folsch UR, Nitsche R, Ludtke R, Hilgers RA, Creutzfeldt W. German study group on acute biliary pancreatitis. N Engl J Med 1997;336:237-42.

53. Takehara $\mathrm{Y}$, Ichijo K, Tooyama N, et al. Breath-hold MR cholangiopancreatography with a long-echo-train fast spin-echo sequence and a surface coil in chronic pancreatitis. Radiology 1994;192:73-8.

54. Soto JA, Barish MA, Yucel EK, et al. MR cholangiopancreatography with a three-dimensional fast spin-echo technique. Radiology 1995;196:459-64

55. Matos C, Metens T, Deviere J, et al. Pancreatic duct: morphologic and functional evaluation with dynamic MR pancreatography after secretin stimulation. Radiology 1997;203:435-41.

56. Soto JA, Yucel EK, Barish MA, Chuttani R, Ferrucci JT. MR cholangiopancreatography after unsuccessful or incomplete ERCP. Radiology 1996;199:91-8.

57. Morgan DE, Baron TH, Smith JK, Robbin ML, Kennet PJ. Pancreatic fluid collections prior to intervention: evaluation with MR imaging compared with CT and US. Radiology 1997;203:773-8.

58. Irie $\mathrm{H}$, Honda $\mathrm{H}$, Shingo $\mathrm{B}$, et al. Autoimmune pancreatitis: $\mathrm{CT}$ and MR characteristics. AJR Am J Roentgenol 1998;170:1323-7.

59. Van Hoe L, Gryspeerdt S, Ectors N, et al. Nonalcoholic duct-destructive chronic pancreatitis: imaging findings. AJR Am J Roentgenol 1998;170:643-7.

60. Mueller MF, Meyenberger C, Bertschonger P, Schaer R, Marincek B. Pancreatic tumors: evaluation with endoscopic US, CT and MR imaging. Radiology 1994;190:745-51.

61. Vellet AD, Romano W, Bach DB, Passi RB, Taves DH, Munk PL. Adenocarcinoma of the pancreatic ducts: comparative evaluation with CT and MR imaging at 1.5 T. Radiology 1992;183:87-95.

62. Irie H, Honda H, Kaneko K, Kuroiwa T, Yoshimitsu K, Masuda K. Comparison of helical CT and MR imaging in detection and 
staging small pancreatic adenocarcinoma. Abdom Imaging 1997;22:429-33.

63. Megibow AJ, Zhou XH, Rotterham H, et al. Pancreatic adenocarcinoma: CT versus MR imaging in the evaluation of resectibility - report of the radiology diagnostic oncology group. Radiology 1995;195:327-32.

64. Semelka RC, Kelekis NL, Molina PL, Sharp TJ, Calvo B. Pancreatic masses with inconclusive findings on spiral CT: is there a role for MRI? J Magn Reson Imaging 1996;6:585-8.

65. Ichikawa T, Haradome H, Hachiya J. Pancreatic ductal adenocarcinoma: preoperative assessment with helical CT versus dynamic MR imaging. Radiology 1997;202:655-62.

66. Catalano C, Pavone P, Laghi A, et al. Pancreatic adenocarcinoma: combination of MR imaging, MR angiography and MR cholangiopancreatography for the diagnosis and assessment of resectibility. Eur Radiol 1998;8:428-34.

67. Trede M, Rumstadt B, Wendl K, et al. Ultrafast magnetic resonance imaging improves the staging of pancreatic tumors. Annal Surg 1997;4:393-407.

68. Smedby O, Riesenfeld V, Karlson BM, et al. Magnetic resonance angiography in the resectibility assessment of suspected pancreatic tumors. Eur Radiol 1997;7:649-53.

69. Adamek HE, Weitz M, Breer H, Jakobs R, Schilling D, Riemann JF. Value of magnetic-resonance cholangio-pancreatography (MRCP) after unsuccessful endoscopic retrograde cholangio-pancreatography (ERCP). Endoscopy 1997;29:741-4.

70. Loperfido S, Angelini G, Benedetti G, et al. Major early complications from diagnostic and therapeutic ERCP: a prospective multicentre trial. Gastrointest Endosc 1998;48:1-10.

71. Becker CD, Grossholz M, Menthna G, de Peyer R, Terrier F. MR cholangiopancreatography: technique, potential indications, and diagnostic features of benign, postoperative and malignant conditions. Eur Radiol 1997;7:865-74.

72. Silverberg E, Boring CE, Squires TS. Cancer statistics, 1990. CA Cancer J Clin 1990;40:9-26.

73. Muto T, Bussey HJR, Morson BC. The evolution of cancer of the colon and rectum. Cancer 1975;36:2251-70.

74. Winawer SJ, Zauber AG, Ho MN, et al. Prevention of colorectal cancer by colonoscopic polypectomy. N Engl J Med 1993;329:1977-81.

75. Winawer SJ, Fletcher RH, Miller L, et al. Colorectal cancer screening: clinical guidelines and rationale. Gastroenterology 1997;112:594-642.

76. Rubin GD, Beaulieu CF, Argiro VA, et al. Perspective volume rendering of CT and MR images: applications for endoscopic imaging. Radiology 1996;199:321-30.

77. Hara AK, Johnson CD, Reed JE, et al. Detection of colorectal polyps by computed tomographic colonography: feasibility of a novel technique. Gastroenterology 1996;110:284-90.

78. Hara AK, Johnson CD, Reed JE, et al. Detection of colorectal polyps with CT colography: initial assessment of sensitivity and specificity. Radiology 1997;205:59-65.

79. Royster AP, Fenlon HM, Clarke PD, Nunes DP, Ferrucci JT. CT Colonoscopy of colorectal neoplasms two-dimensional and three-dimensional virtual-reality techniques with colonoscopic correlation. AJR Am J Roentgenol 1997;169:1237-42.

80. McFarland EG, Brink JA, Loh J, et al. Visualization of colorectal polyps with spiral CT colography: evaluation of processing parameters with perspective volume rendering. Radiology 1997;205:701-7.

81. Dachman AH, Kuniyoshi JK, Boyle CM, et al. CT colonography with three-dimensional problem solving for detection of colonic polyps. AJR Am J Roentgenol 1998;171:989-95.

82. McFarland EG, Wang G, Brink JA, et al. Spiral CT colonography: central axis determination and digital unraveling of the colon. Acad Radiol 1997;4:367-73.

83. Kay CL, Evangelou HA. A review of the technical and clinical aspects of virtual endoscopy. Endoscopy 1996;28:768-75.

84. Vining DJ, Hemler PF, Stelts DR, et al. Virtual endoscopy: quicker and easier disease evaluation. SPIE 1997;3033:415-23.

85. Dachman AH, Lieberman J, Osnis RB, et al. Small simulated polyps in pig colon: sensitivity of virtual colography. Radiology 1997;203:427-30.

86. Beaulieu CF, Napel S, Daniel BL, et al. Detection of colonic polyps in a phantom model: implications for virtual colonoscopy data acquisition. J Comput Assist Tomogr 1998;22:656-63.

87. Paik DS, Beaulieu CF, Jeffrey RB, Rubin GD, Napel S. Automated flight path planning for virtual endoscopy. Med Phys 1998;25:629-37.

88. Vining DJ. Optimizing bowel preparation. First International Symposium on Virtual Colonoscopy, Boston, October, 1998.

89. Wang G, McFarland EG, Brown BP, Vannier MW. GI Tract Unraveling with Curved Cross-Sections. IEEE Trans Med Imaging 1998;17:318-22.

90. Paik DS, Beaulieu CF, Jeffrey RB, Karadi CA, Napel S. Panoramic virtual colonoscopy using the Mercator projection. First International Symposium on Virtual Colonoscopy, Boston, October, 1998. 


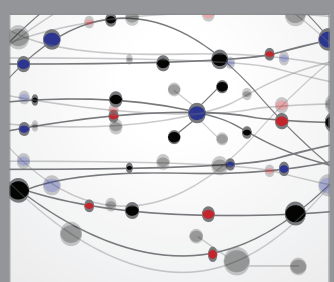

The Scientific World Journal
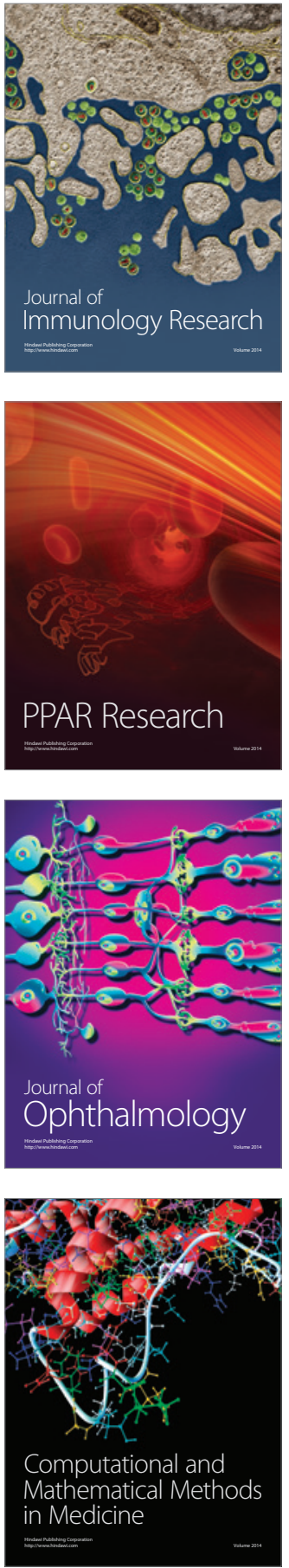

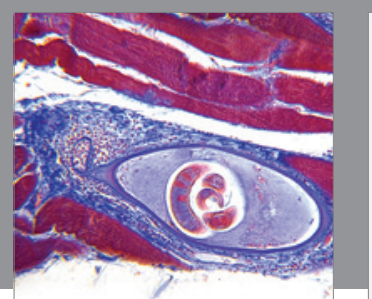

Gastroenterology Research and Practice

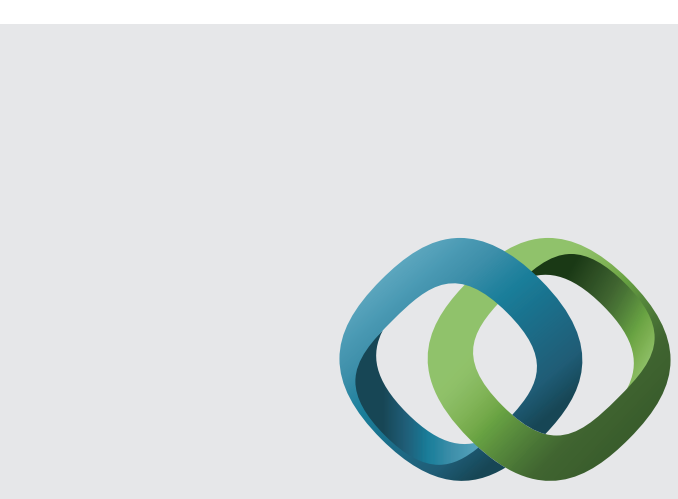

\section{Hindawi}

Submit your manuscripts at

http://www.hindawi.com
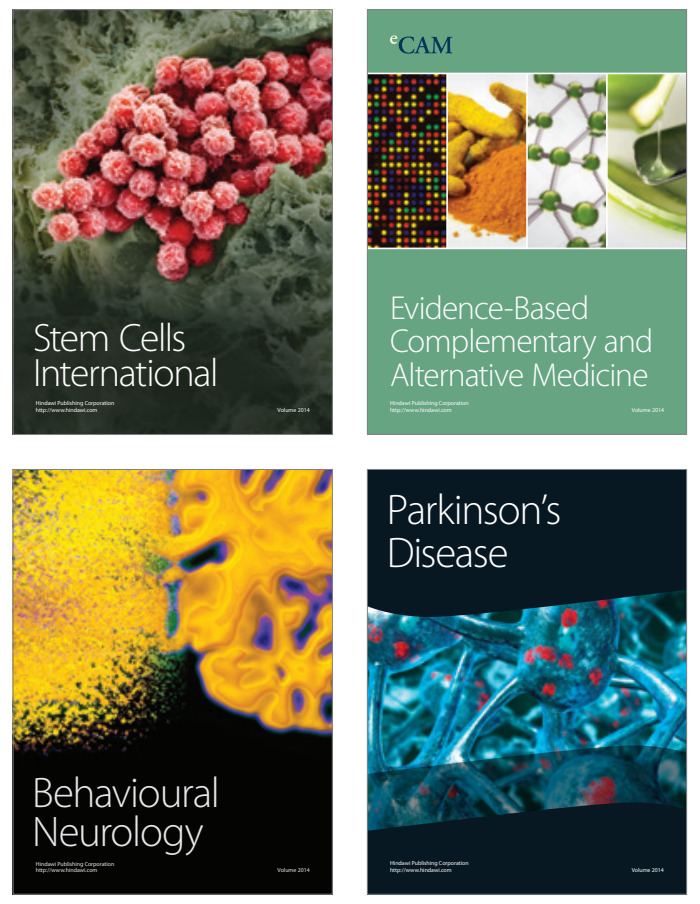
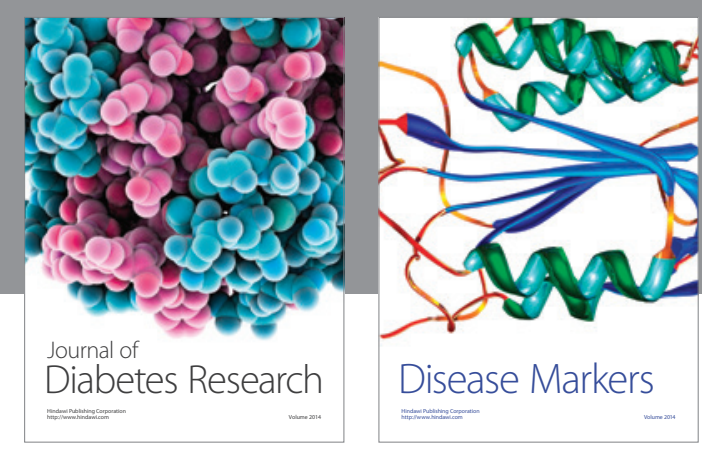

Disease Markers
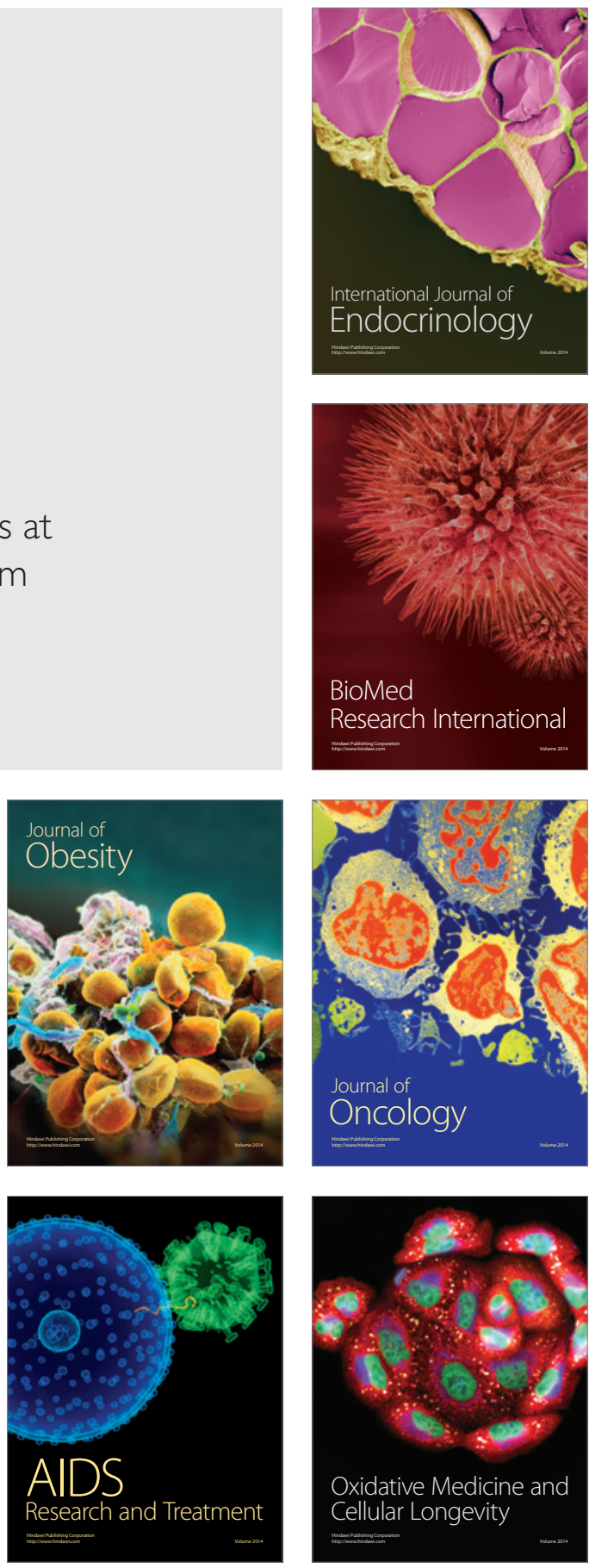